CERN-TH/99-97

\title{
Quantum Irreversibility in Arbitrary Dimension
}

\author{
Damiano Anselmi \\ CERN, Division Théorique, CH-1211, Geneva 23, Switzerland
}

\begin{abstract}
Some recent ideas are generalized from four dimensions to the general dimension $n$. In quantum field theory, two terms of the trace anomaly in external gravity, the Euler density $\mathrm{G}_{n}$ and $\square^{n / 2-1} R$, are relevant to the problem of quantum irreversibility. By adding the divergence of a gauge-invariant current, $\mathrm{G}_{n}$ can be extended to a new notion of Euler density $\tilde{\mathrm{G}}_{n}$, linear in the conformal factor. We call it pondered Euler density. This notion relates the trace-anomaly coefficients $a$ and $a^{\prime}$ of $\mathrm{G}_{n}$ and $\square^{n / 2-1} R$ in a universal way $\left(a=a^{\prime}\right)$ and gives a formula expressing the total RG flow of $a$ as the invariant area of the graph of the beta function between the fixed points. I illustrate these facts in detail for $n=6$ and check the prediction to the fourth-loop order in the $\varphi^{3}$-theory. The formula of quantum irreversibility for general $n$ even can be extended to $n$ odd by dimensional continuation. Although the trace anomaly in external gravity is zero in odd dimensions, I show that the odd-dimensional formula has a predictive content.
\end{abstract}

CERN-TH/99-97 - April, 1999 


\section{Introduction}

The trace anomaly is a useful tool to study non-perturbative properties of quantum field theory making use of perturbation theory only. A particularly fruitful arena for this kind of investigation is the conformal window, where the renormalization-group $(\mathrm{RG})$ flow interpolates between two conformal field theories; this interpolation is understood in the sense of the resummation of the perturbative expansion, even when the interacting fixed point is strongly coupled.

Embedding the theory in external gravity is particularly convenient to study the RG interpolation between the UV and IR values of the so-called central charges, i.e. the coefficients $c$, $a$ and $a^{\prime}$ of the gravitational invariants appearing in the trace anomaly.

The central charges satisfy positivity conditions and the spectra of anomalous dimensions obey even stronger restrictions [1, 2], which give non-trivial information about the low-energy limit of the theory. Other inequalities are strictly related to unitarity, such as the irreversibility of the RG flow, which is the statement that $a_{\mathrm{UV}} \geq a_{\mathrm{IR}}$.

In [3] we studied the problem of quantum irreversibility in four dimensions. A non-perturbative formula expressing the total $a$-flow as the invariant area of the graph of the beta function between the fixed point comes out naturally. This formula agrees with perturbation theory up to the fourth-loop order included. Moreover, a natural positivity property of the induced action for the conformal factor implies the $a$-theorem to all orders, i.e. the inequalities $a_{\mathrm{UV}} \geq a_{\mathrm{IR}} \geq 0$ and the formula for $\Delta a=a_{\mathrm{UV}}-a_{\mathrm{IR}}$.

Precisely, it was explained in [3] that quantum irreversibility is measured by the invariant area of the graph of the beta function between the fixed points. In particular, there exists a one-form

$$
\omega=-\mathrm{d} \lambda \beta(\lambda) f(\lambda),
$$

where $f(\lambda) \geq 0$ is a metric in the space of coupling constants, defined by

$$
\langle\Theta(x) \Theta(0)\rangle=\frac{1}{15 \pi^{4}} \frac{\beta^{2}(t) f(t)}{|x|^{8}}
$$

in four dimensions $(t=\ln |x| \mu)$. $\Theta$ is the trace of the stress-tensor. The ideas of [3] can be summarized by the formula

$$
\Delta a=\Delta a^{\prime}=\int_{\mathrm{UV}}^{\mathrm{IR}} \omega \geq 0,
$$

saying that the total RG flow of $a$ is the integral of the form $\omega$ between the fixed points. This statement was checked to the fourth-loop order in the most general renormalizable fourdimensional theory.

It is therefore interesting to understand whether the new approach extends directly to arbitrary dimension, or further obstructions, similar to the conceptual gap between irreversibility in two- and four-dimensions, make this task less straightforward.

However, the motivation for exploring higher-dimensional field theories is not purely academic. The final aim is to extend the study of quantum irreversibility, well-established in two 
and four dimensions, to three-dimensional quantum field theory and phenomena relevant to condensed matter physics, for instance superconductivity.

The trace anomaly is an intrinsic effect of renormalization, in particular a non-vanishing beta function [4, 5, 6], and exists in every dimension. For example, $\Theta$ is $\frac{\beta(\lambda)}{6 !} \varphi^{6}$ in the $\varphi^{6}$ theory in three dimensions. The anomaly begins at two loops instead of one (because odd-loop Feynman graphs do not diverge in odd dimensions), which is the reason why it is not visible as an ordinary one-loop anomaly.

The trace anomaly operator equation in flat space is not sufficient in itself to study the phenomenon of quantum irreversibility. One needs the trace anomaly in the presence of an external background (gravity in even dimensions) that supports certain invariants (such as the Euler density) and therefore appropriate anomalies, the central charges, in particular the quantities $a$ and $a^{\prime}$. There is no such background in odd dimensions. Therefore, we do not see any other way of extending the ideas to odd dimensions than continuing those formulas that hold in even dimensions. From the formal point of view, this does not present problems. The coefficients to be continued to odd dimensions are indeed very simple functions of the dimension $n$. I will discuss a situation in which the odd-dimensional formula can in principle be checked.

This is, in summary, the reason why it is interesting to treat the problem of generic $n$. I start by presenting some aspects of the ideas of [3] from the point of view of differential geometry, to clarify how the generalization to arbitrary $n$ can be achieved. I stress, however, that the major source of inspiration for these ideas is physics. Many identities could be found only with a major effort, if they were not inspired by the physical ideas behind them.

For definiteness, in the first part of the paper I focus on $n=6$, or $n=6-\varepsilon$. Several identities hold for arbitrary $n$, a fact that is quite important to reduce the number of independent terms in the regularized theory. A special section is devoted to the generalization of the irreversibility formula to arbitrary dimension.

The Gauss-Bonnet integrand, or Euler density,

$$
\mathrm{G}_{6}=-\varepsilon^{\mu \nu \rho \sigma \tau \xi} \varepsilon_{\alpha \beta \gamma \delta \epsilon \zeta} R_{\mu \nu}^{\alpha \beta} R_{\rho \sigma}^{\gamma \delta} R_{\tau \xi}^{\epsilon \zeta},
$$

is a non-trivial total derivative, i.e. the total derivative of a non-gauge-covariant current (the Chern-Simons form). It is clear, then, that $\mathrm{G}_{6}$ is defined up to trivial total derivatives, i.e. up to the divergence of a gauge-covariant current. The topological numbers calculated with any modified Gauss-Bonnet integrand of the form $\tilde{\mathrm{G}}_{6}=\mathrm{G}_{6}+\nabla_{\alpha} J^{\alpha}$ are exactly the same as those computed with the ordinary one. The modified integrand, however, can be chosen so as to have an additional remarkable property, namely to be linear in the conformal factor. The anomaly equation and the induced action for the conformal factor simplify enormously. The modified integrand will be called pondered Euler density and is meaningful in every even dimension.

This phenomenon is already apparent in four dimensions, where the pondered Euler density is

$$
\tilde{\mathrm{G}}_{4}=\mathrm{G}_{4}-\frac{8}{3} \square R=\mathrm{G}_{4}+\nabla_{\alpha} J_{4}^{\alpha}, \quad J_{4}^{\alpha}=-\frac{8}{3} \nabla_{\alpha} R .
$$

\footnotetext{
${ }^{1}$ In the notation of the present paper $\mathrm{G}_{4}$ is 4 times the Gauss-Bonnet integrand of ref. [3]. See (5.29).
} 
According to ref. [3] the conceptual gap between quantum irreversibility in two [7] and four dimensions is filled by the pondered extension of the Euler density. This is the true Euler density that should appear in the trace anomaly operator equation, thereby removing the ambiguities associated with the trivial total derivative terms appearing in the trace anomaly. The higherdimensional extension of these ideas requires no other conceptual innovation.

In higher dimensions the possibility of fine-tuning the coefficients of the numerous additional terms to construct a pondered Euler density is far more upsetting than in four dimensions, in view of the complexity of the expressions.

Inspired by these ideas, we have found that in six dimensions the combination

$$
\sqrt{g} \tilde{\mathrm{G}}_{6} \equiv \sqrt{g}\left[\mathrm{G}_{6}+\nabla_{\alpha} J_{6}^{\alpha}\right]=48 \square^{3} \phi
$$

is indeed linear in the conformal factor $\phi$ for conformally flat metrics $g_{\mu \nu}=\mathrm{e}^{2 \phi} \delta_{\mu \nu}$, if

$$
\begin{aligned}
J_{6}^{\alpha}= & -\left(\frac{408}{5}-20 \zeta\right) R_{\mu}^{\nu} \nabla_{\nu} R^{\mu \alpha}-\left(\frac{36}{25}+2 \zeta\right) R^{\alpha \mu} \nabla_{\mu} R \\
& +\zeta \nabla^{\alpha} R^{2}+\left(\frac{144}{5}-10 \zeta\right) \nabla^{\alpha}\left(R_{\mu \nu} R^{\mu \nu}\right)-\frac{24}{5} \nabla^{\alpha} \square R .
\end{aligned}
$$

We do not need to use the Riemann tensor $R_{\mu \nu \rho \sigma}$. We re-express it as a combination of the Ricci tensor $R_{\mu \nu}$ and the scalar curvature $R$, plus the Weyl tensor $W_{\mu \nu \rho \sigma}$. Most of our work in this paper focuses on the trace $\Theta$ of the stress-tensor, coupled to the conformal factor $\phi$. It is understood that our formulas are written "up to terms proportional to the Weyl tensor".

Our requirement (1.5) does not seem to fix $J_{6}^{\alpha}$ uniquely, but leaves a free parameter $\zeta$. We do not need $\zeta$, since $\zeta$ does not affect the term $\square^{2} R$, but this parameter is the sign of a redundancy in our list of terms. Exploring this issue better, we find that the combination

$$
\nabla_{\alpha}\left[\nabla^{\alpha} R^{2}-2(n-1) \nabla^{\alpha}\left(R_{\mu \nu} R^{\mu \nu}\right)-2 R^{\alpha \mu} \nabla_{\mu} R+4(n-1) R_{\mu}^{\nu} \nabla_{\nu} R^{\mu \alpha}\right]
$$

is identically zero on conformally-flat metrics in arbitrary dimension $n$. The fact that this is true for arbitrary $n$ and not just for $n=6$ allows us to consistently reduce the number of terms, in a regularized theory as well. We choose $\zeta=\frac{102}{25}$, so that

$$
J_{6}^{\alpha}=-\frac{48}{5} R^{\alpha \mu} \nabla_{\mu} R+\frac{102}{25} \nabla^{\alpha} R^{2}-12 \nabla^{\alpha}\left(R_{\mu \nu} R^{\mu \nu}\right)-\frac{24}{5} \nabla^{\alpha} \square R .
$$

Writing $\sqrt{g} \mathrm{G}_{6}=\partial_{\alpha} C^{\alpha}$, where $C^{\alpha}$ is the Chern-Simons form, the pondered Chern-Simons form reads $\tilde{C}^{\alpha}=C^{\alpha}+\sqrt{g} J^{\alpha}$ and $\sqrt{g} \tilde{\mathrm{G}}_{6}=\partial_{\alpha} \tilde{C}^{\alpha}$.

In ref. [8] Karakhayan et al. have explicitly written a conformal-invariant operator of the form $\square^{3}+$ curvature terms in six dimensions. A similar operator (of the form $\square^{2}+$ curvature terms) exists in four dimensions [9]. It is actually the variation of the pondered Euler density with respect to the conformal factor, and it can be read in six dimensions by varying $\tilde{\mathrm{G}}_{6}$ under a general rescaling $g_{\mu \nu} \rightarrow \mathrm{e}^{2 \phi} g_{\mu \nu}$. $\tilde{\mathrm{G}}_{6}$ can also be derived from the results of ref. [8], with a certain amount of work. The pondered Euler density is the basic ingredient to write down the higher-dimensional analogue of the Riegert action [9]; it is unique up to terms proportional to 
the Weyl tensor. In section 5 the expression of $\tilde{\mathrm{G}}_{8}$ is reported and the construction for arbitrary even $n$ is presented.

The notion of pondered Euler density relates in a universal way the trace-anomaly coefficients of $\mathrm{G}_{6}$ and $\square^{2} R$, which we can call $a$ and $a^{\prime}$, respectively. It allows us to generalize the formula of [3], measuring the effect of quantum irreversibility in terms of the area of the graph of the beta function between the fixed points (section 2).

The rest of the paper is organized as follows. After a detailed classification of the curvature terms occurring in the bare lagrangian and in the trace anomaly (section 3), we check this prediction to the fourth-loop order in perturbation theory for the $\varphi^{3}$-theory in six dimensions (section 4); this is a good opportunity to show that the RG equations produce exactly the pondered Euler density, eliminating step by step the $\phi$-interaction terms in the trace anomaly. The $a$-theorem can indeed be seen as a non-renormalization theorem for the $\Theta$ many-point functions. The general formula is extended to arbitrary $n$ in section 5 , where the continuation to odd dimensions is discussed, together with a suggestion for a check.

Before beginning the technical study, we make a comment of a general character. Quantum irreversibility is intrinsically related to the beta function, as we have stressed. The beta function plays a major role in the RG interpolation problem. Now, perturbation theory cannot be applied too naively in this context, otherwise fake ambiguities and sometimes wrong conclusions do arise. There is a well-known scheme change, proposed by 't Hooft [10], in which the beta function is just two-loop. If this were consistent in the RG-interpolation problem, we could conclude immediately that the $\varphi^{4}$-theory, say, has a UV interacting fixed point, that pure Yang-Mills theory has no IR fixed point, etc. However, the scheme change under consideration is allowed only order by order in perturbation theory.

The point is that an apparently harmless power series in $\alpha$ can resum to a function proportional to the inverse of the beta function. An example is the function $\alpha / \beta$ in $\mathrm{QCD}$, where $\beta=\mathrm{d} \ln \alpha / \mathrm{d} \ln \mu$. A scheme change generated by such a function is admissible in the naive sense, not in our problem, since it spoils a proper interpolation between the fixed points. The moral of the story is that one has to keep track of the orders of $\beta$ and $\alpha$, not only of the powers of $\alpha$ ?.

The appearance of orders of $\beta$ can often be rigorously detected and a good approach to the $\mathrm{RG}$ interpolation problem relies on this fact. An illustration is provided precisely by the trace anomaly, whose existence was first realized by Coleman and Jackiw in ref. [13]. It was later shown [4] that the general form of the trace anomaly reads $\Theta=\beta \mathrm{O}$, O being a certain operator ( $F^{2} / 4$ in the case of QED and Yang-Mills theory). While both $\beta$ and $\mathrm{O}$ depend on the scheme, the invariant content of this equation is precisely that $\Theta$ is of order $\beta$ around the fixed points and that a scheme change that spoils this property is not admissible. This is in agreement with the restored conformal invariance at the fixed points.

Sometimes, axial anomalies are proportional to the beta function also. In particular, this applies, in supersymmetric theories, to the divergence of the $R$-current, which is the supersym-

\footnotetext{
${ }^{2}$ These ideas have been discussed and applied extensively in 11, 12, to which we refer the readers for further details.
} 
metric partner of $\Theta$. There is no contradiction with the Adler-Bardeen theorem, since currents differring by $\mathcal{O}(\epsilon)$-terms (we assume that we are working in dimensional regularization) can well satisfy different anomaly equations. In one case the divergence has the form predicted by the Adler-Bardeen theorem, in another case it is proportional to the beta function [14]. In agreement with our observations, the scheme change that interchanges the two anomaly equations is singular (see for example ref. [15]). The important thing for the applications [12] is to identify which current is the correct partner of $\Theta$ (this problem has been known for some time as the anomaly puzzle).

When embedding the theory in external gravity, the careful analysis of [17, 18], which we are going to use extensively in this paper, identifies other places where orders of $\beta$ appear. Most scheme dependences in the central charges are proportional to the beta function 11] and therefore do not affect the critical values and the total RG flows. These techniques allow us to show that the renormalization-group equations select precisely the combination of gravitational invariants that we have called pondered Euler density.

In summary, quantum field theory defines a natural fibre bundle, that we call scheme bundle. The base manifold is the space of physical correlators and the fibre is the space of allowed scheme choices, with the regularity restriction outlined above. A projection onto the base manifold is defined and assures scheme independence of the physical correlators. The scheme bundle is equipped with a metric $f$ and a fundamental one-form $\omega$, see (1.1). In integrals expressing total $\mathrm{RG}$ flows, such as (1.2), scheme independence is reparametrization invariance. The bundle admits a "proper" section (and so a proper beta function and a proper coupling constant), defined as the scheme choice for which the metric $f$ is constant throughout the RG flow (see [3] for the detailed construction).

This geometric approach to quantum field theory is complementary to a similar notion introduced in [19] for the algorithm of subtraction of divergences in the most general gauge field theory. In that case a fibre bundle was defined, whose fibre was the space of fields and antifields and whose base manifold was, again, the space of physical parameters and correlators. The removal of divergences was proved to be the direct product of a diffeomorphism in the base manifold and a canonical transformation on the fibre. There is a natural structure of connections and curvatures that helps the generalization of the known theorems of renormalization theory.

\section{Idea and prediction}

At criticality the trace anomaly reads

$$
\Theta=a_{*} \mathrm{G}_{6}+\text { conformal invariants }+ \text { trivial divergences. }
$$

For a free theory with $N_{s}$ real scalar fields, $N_{f}$ Dirac fermions and $N_{v}$ 2-forms, we have

$$
a_{\text {free }}=\left(N_{s}+f_{6} N_{f}+v_{6} N_{v}\right) \text { const. }
$$

Results for $a_{\text {free }}$, in particular the overall constant and the relative factor $f_{6}$, can be found in ref. [20]. 
The idea of [3] is that the "trivial" divergences are fixed uniquely by the property (1.5) and in the end are not so trivial as is commonly assumed, since they produce a precise formula for the effect of quantum irreversibility. From the point of view of explicit calculations, these terms contain a certain type of ambiguity, which makes them ill-defined by arbitrary additive finite bare parameters. Nevertheless, the ambiguities can be consistently removed in a universal way, writing

$$
\Theta=a_{*} \tilde{\mathrm{G}}_{6}+\text { conformal invariants. }
$$

The two-point function of $\Theta$ is proportional to the number $a_{*}$ in the conformal limit $\beta=0$. We get

$$
\langle\Theta(x) \Theta(y)\rangle=-\frac{\delta^{2} S_{\mathrm{E}}[\phi]}{\delta \phi(x) \delta \phi(y)} \mid=-48 a_{*} \square^{3} \delta(x-y) .
$$

Here $S_{\text {eff }}[\phi]$ denotes the induced effective action for the conformal factor. The notation $Q \mid$ means that the quantity $Q$ is evaluated in flat space. Formula (2.1) gives, in the case of a conformally-flat metric,

$$
\Theta=48 a_{*} \square^{3} \phi
$$

and the induced action is

$$
S_{\mathrm{E}}=-24 a_{*} \int \mathrm{d}^{6} x\left(\square \partial_{\mu} \phi\right)^{2} .
$$

With the identification $a^{\prime}=a$, i.e. with the modified Gauss-Bonnet integrand, the $\phi$-action is free at criticality, which means that the three- and many-point functions of $\Theta$ are zero. In four dimensions the two-point function $\langle\Theta(x) \Theta(0)\rangle$ equals $-\frac{1}{90(4 \pi)^{2}} a \square^{2} \delta(x)$. In two dimensions it is $\langle\Theta(x) \Theta(0)\rangle=-\frac{\pi}{3} c \square \delta(x)$.

Using formula (2.4) we see that the quantity $a_{*}$ can be expressed at criticality by the integral

$$
a_{*}=-\frac{1}{2^{13} \cdot 3^{3} \cdot 5} \int \mathrm{d}^{6} x|x-y|^{6}\langle\Theta(x) \Theta(y)\rangle .
$$

We now consider the off-critical theory. We can define a function $a^{\prime}(r)$ of the intermediate energy scale $1 / r$ by restricting the integration over a four-sphere $S(r, y)$ of radius $r$ and centred at the point $y$, precisely

$$
a\left(r_{2}\right)-a\left(r_{1}\right)=-\frac{1}{2^{13} \cdot 3^{3} \cdot 5} \int_{S\left(r_{1}, y\right)}^{S\left(r_{2}, y\right)} \mathrm{d}^{6} x|x-y|^{6}\langle\Theta(x) \Theta(y)\rangle .
$$

For a critical theory we have $a_{\mathrm{UV}}=a_{\mathrm{IR}}=a(r)=a_{*}$. Off-criticality, the running of $a^{\prime}(r)$ is due to the internal term (e.g. $\frac{\beta}{3 !} \varphi^{3}$ in the $\varphi^{3}$-theory) appearing in the expression of $\Theta$. There is a non-local contribution to the correlator $\langle\Theta(x) \Theta(y)\rangle$,

$$
\langle\Theta(x) \Theta(y)\rangle=\frac{2^{13} \cdot 3^{3} \cdot 5}{\pi^{3}} \frac{\beta^{2}[\lambda(t)] f[\lambda(t)]}{|x-y|^{12}}, \quad \text { for } x \neq y,
$$

so that the total flow of the quantity $a$ is non-negative and equal to the invariant area of the beta function:

$$
a_{\mathrm{UV}}-a_{\mathrm{IR}}=\int_{-\infty}^{+\infty} \mathrm{d} t \beta^{2}(t) f(t)=-\int_{\lambda_{\mathrm{UV}}}^{\lambda_{\mathrm{IR}}} \mathrm{d} \lambda \beta(\lambda) f(\lambda) \geq 0
$$


This prediction can be checked to the fourth-loop order in perturbation theory. We will consider the $\varphi^{3}$-theory in six dimensions. This theory is not a meaningful physical theory, but it is the only renormalizable theory in dimension greater than four. It was observed in [3] that formula (2.8) is expected to work even in the absence of an interacting fixed point and can be consistently checked order by order in perturbation theory whenever the perturbative expansion makes sense. The price is that we cannot demand that the inequality $a_{\mathrm{UV}} \geq a_{\mathrm{IR}}$, which is the natural generalization of Cardy's conjecture [21] to six dimensions, be satisfied.

In our notation the lagrangian is

$$
\int \mathcal{L}=\int \mathrm{d}^{6} x\left[\frac{1}{2}\left(\partial_{\mu} \varphi^{i}\right)^{2}+\lambda \varphi_{1} \varphi_{2} \varphi_{3}\right]
$$

where $i=1,2,3$. We choose this form so as to avoid counterterms linear in $\varphi$. We sometimes compactify the notation by writing $\mathcal{L}=\frac{1}{2}\left(\partial_{\mu} \varphi\right)^{2}+\frac{\lambda}{3 !} \varphi^{3}$, but it should be kept in mind that what we mean is the theory (2.9).

We follow the procedure of [17], which we generalize to six dimensions. It was explained in [3] that we do not need, to the order we are interested in, to worry about the non-minimal term $R \varphi^{2}$ in the extension of (2.9) to curved space. The two-loop beta function is [22]

$$
\beta(\lambda)=-\frac{1}{2} \frac{\lambda^{3}}{(4 \pi)^{3}}-\frac{41}{36} \frac{\lambda^{5}}{(4 \pi)^{6}}+\mathcal{O}\left(\lambda^{7}\right) \equiv \beta_{1} \lambda^{3}+\beta_{2} \lambda^{5}+\mathcal{O}\left(\lambda^{7}\right)
$$

The theory is formally asymptotically free. We have $\Theta=-\beta(\lambda) \varphi_{1} \varphi_{2} \varphi_{3}$ and therefore

$$
f_{*}=\frac{\pi^{3}}{2^{13} \cdot 3^{3} \cdot 5}|x|^{12}\left(\left\langle\varphi_{1}(x) \varphi_{1}(0)\right\rangle\right)^{3}=\frac{1}{2^{7} \cdot 3^{3} \cdot 5(4 \pi)^{6}}
$$

since the $\varphi$-propagator is $\langle\varphi(x) \varphi(0)\rangle=-1 / \square=1 /\left(4 \pi^{3}\right) 1 /|x|^{4}$.

As was explained in ref. [3], our formula predicts the $a$-flow even in the absence of a rigorous fixed point. However, in order to achieve this goal, we have to pretend that a fixed point does indeed exist. This can be done as follows. We write $\beta(\lambda)=\beta_{2} \lambda^{3}\left(\lambda^{2}-\lambda_{\mathrm{IR}}^{2}\right)+\mathcal{O}\left(\lambda^{7}\right)$ and pretend that $\lambda_{\mathrm{IR}}^{2}=-\beta_{1} / \beta_{2}$ is a small parameter. In other words, we consider $\beta_{1}$ as an independent, small parameter, and replace it with $-\beta_{2} \lambda_{\mathrm{IR}}^{2}$ everywhere. This procedure is unambiguous as long as we can keep track of the orders of $\beta$, not only the orders of $\alpha$, as explained in the introduction. We will see that there is enough information to carry this over to the end. Of course, there would be no such nuisance in the presence of a true zero of the beta function.

We therefore have, to the lowest order:

$$
a_{\mathrm{UV}}-a\left(\lambda_{\mathrm{IR}}\right)=-\int_{0}^{\lambda_{\mathrm{IR}}} d \lambda^{\prime} f_{*} \beta_{2} \lambda^{\prime 3}\left(\lambda^{\prime 2}-\lambda_{\mathrm{IR}}^{2}\right)=\frac{1}{12} \beta_{2} f_{*} \lambda_{\mathrm{IR}}^{6}
$$

and the fourth-loop-order prediction

$$
a(\lambda)=a_{\text {free }}-\frac{1}{12} \beta_{2} f_{*} \lambda^{6}+O\left(\lambda^{8}\right)=a_{\text {free }}+\frac{41}{7464960} \frac{\lambda^{6}}{(4 \pi)^{12}}+O\left(\lambda^{8}\right),
$$


or

$$
\Theta=\left(a_{\text {free }}+\frac{41}{7464960} \frac{\lambda^{6}}{(4 \pi)^{12}}\right) \mathrm{G}_{6}+\text { rest. }
$$

Note that the check of $\Delta a$ is independent of the value $a_{\text {free }}$. This value is needed to normalize $a$ conventionally, but Hathrell's techniques, which we extend here to six dimensions, give the flow $\Delta a$ directly and are actually unable of predicting the value $a_{\text {free }}$. We stress again that the first relevant radiative correction is fourth-loop in our case, while it is generically third-loop in four dimensions.
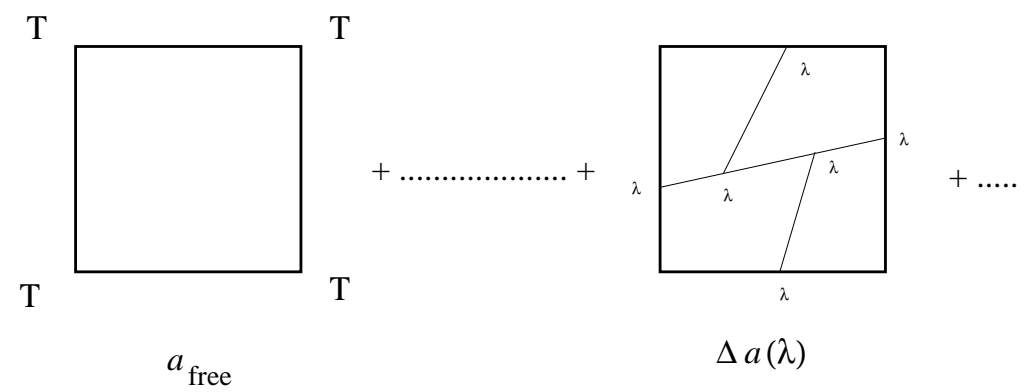

$a_{\text {free }}$

$\Delta a(\lambda)$

Fig. 1: the direct computation of $\Delta a(\lambda)$ would be four-loop.

\section{Classification of the curvature terms}

In this section we discuss the general structure of the trace anomaly and the bare lagrangian. A detailed introduction to the subject can be found in ref. [23].

The conformal-invariant terms are four. Two terms are cubic in the Weyl tensor,

$$
A_{1}=W_{\mu \nu \rho \sigma} W^{\mu \nu \alpha \beta} W_{\alpha \beta}^{\rho \sigma}, \quad A_{2}=W_{\mu \nu \rho \sigma} W^{\mu \alpha \rho \beta} W_{\alpha}{ }^{\nu}{ }^{\sigma} .
$$

A third term has the form

$$
A_{3}=W_{\mu \nu \rho \sigma} \square W^{\mu \nu \rho \sigma}+\cdots
$$

and is directly related to the central extension $c$ of the OPE algebra (see [11] for details):

$$
\left\langle T_{\mu \nu}(x) T_{\rho \sigma}(0)\right\rangle=c \prod_{\mu \nu, \rho \sigma}^{(2)} \square\left(\frac{1}{|x|^{6}}\right),
$$

$T_{\mu \nu}$ denoting the stress-tensor and $\prod_{\mu \nu, \rho \sigma}^{(2)}$ being the spin-2 projector (see for example [24]). To see this, we use the relations

$$
\mu \frac{\partial}{\partial \mu} \rightarrow \int \Theta, \quad \mu \frac{\partial}{\partial \mu} \frac{1}{|x|^{6}}=\pi^{3} \delta(x)
$$

so that

$$
\frac{\delta^{2}}{\delta g_{\mu \nu}(x) \delta g_{\mu \nu}(0)} \int \Theta \mid=\left\langle\int \Theta T_{\mu \nu}(x) T_{\rho \sigma}(0)\right\rangle \rightarrow \pi^{3} c \prod_{\mu \nu, \rho \sigma}^{(2)} \square \delta(x)
$$


and therefore $\Theta \sim c A_{3}+$ rest. The central-extension term reads $W_{\mu \nu \rho \sigma} \square^{n / 2-2} W^{\mu \nu \rho \sigma}+\cdots$ in generic even dimension $n>2$.

The complete form of $A_{3}$ that can be found in the literature (for example, in ref. [ 8 , formula (2.18)) is not explicitly proportional to the Weyl tensor. This is because a spurious contribution proportional to $\mathrm{G}_{6}$ is included. It is easy to see, however, that subtracting this term away, $A_{3}$ can be re-expressed in a form that is proportional to the Weyl tensor (see for example [16]). This fact shows that $A_{3}$ is negligible for our purposes, as well as $A_{1}$ and $A_{2}$.

In conclusion, there is only one term that is relevant to our analysis, and this is $\mathrm{G}_{6}$.

The most general bare lagrangian contains in the gravity sector the following terms

$$
\mathcal{L}_{\mathrm{B}}=a_{\mathrm{B}} \mathrm{G}_{6}+b_{\mathrm{B}} R R_{\mu \nu} R^{\mu \nu}+c_{\mathrm{B}} R^{3}+d_{\mathrm{B}} R \square R+e_{\mathrm{B}} R_{\mu \nu} \square R^{\mu \nu},
$$

where $\mathrm{G}_{6}(1.3)$ is continued to dimension $n$ as

$$
-12 R_{\mu \nu} R^{\nu \rho} R_{\rho}^{\mu}+\frac{9 n}{n-1} R R_{\mu \nu} R^{\mu \nu}-\frac{3\left(n^{2}+4 n-4\right)}{4(n-1)^{2}} R^{3}+\mathcal{O}(W),
$$

and terms proportional to the Weyl tensor $W$, irrelevant to our analysis, are omitted.

In the above list, there is one redundancy, as a reflection of the property noted in (1.6) at the level of total derivatives. Indeed the combination

$$
\begin{aligned}
& -2(n-1) R_{\mu \nu} \square R^{\mu \nu}-\frac{n}{2}\left(\nabla_{\mu} R\right)^{2}+\frac{1}{2} \square R^{2}+(n-2) \nabla_{\mu}\left(R^{\mu \nu} \nabla_{\nu} R\right) \\
& +2 \frac{n(n-1)}{n-2} R_{\mu \nu} R^{\nu \rho} R_{\rho}^{\mu}-2 \frac{2 n-1}{n-2} R R_{\mu \nu} R^{\mu \nu}+\frac{2}{n-2} R^{3}
\end{aligned}
$$

is identically zero on conformally flat metrics, in arbitrary dimension $n$. The derivative of the above expression (integrated over the space-time) with respect to the conformal factor is $(n-6)$ times the same expression. For this reason, it is negligible for our purposes. Therefore we consistently remove the parameter $e_{\mathrm{B}}$ in (3.2).

In the end we remain with $\mathrm{G}_{6}$ and the three terms

$$
R R_{\mu \nu} R^{\mu \nu}, \quad R^{3}, \quad R \square R,
$$

at the level of the bare lagrangian. All the other terms are either proportional to the Weyl tensor or total derivatives in $n$ dimensions and can therefore be omitted. The three terms (3.4) are forbidden at criticality, since their integrals are not conformal-invariant in $n=6$. Off-criticality, they do appear, but their coefficients, proportional to the beta function, will be related to $a_{\mathrm{B}}$ in a way that we have to uncover (and that should, in particular, agree with our prediction $\left.\Delta a=\Delta a^{\prime}\right)$. There is some surviving ambiguity, actually, since $b_{\mathrm{B}}, c_{\mathrm{B}}$ and $d_{\mathrm{B}}$ can be shifted by arbitrary additive constants.

The same three terms appear, multiplied by $(n-6)$, in the trace anomaly equation. Moreover, four independent total-derivative terms,

$$
\square\left(R_{\mu \nu} R^{\mu \nu}\right), \quad \square R^{2}, \quad \nabla_{\mu}\left(R^{\mu \nu} \nabla_{\nu} R\right), \quad \square^{2} R,
$$


appear at the level of the trace anomaly $\Theta$, with coefficients related to the coefficients $a_{\mathrm{B}}, b_{\mathrm{B}}$, $c_{\mathrm{B}}$ and $d_{\mathrm{B}}$ (the coefficients of these terms in $\Theta$ do not come multiplied by $(n-6)$ ).

It is worth recalling that in four dimensions we have the terms $\mathrm{G}_{4}$ and $R^{2}$ at the level of the bare lagrangian, $\mathrm{G}_{4}, R^{2}$ and $\square R$ at the level of the trace anomaly. The integrability condition requires that the coefficient of $R^{2}$ in the renormalized lagrangian be proportional to the beta function and so vanish at criticality. The coefficient of $\square R$ in $\Theta$ is directly related to the coefficient of $R^{2}$.

\section{Perturbative calculation}

In this section I apply Hathrell's techniques to the $\varphi^{3}$-theory in six dimensions, following the notation of [17] (turned to the Euclidean framework) as close as possible in order to facilitate the reading. There are nevertheless unavoidable complications due to the large number of invariants. I take the opportunity, in the derivation, to stress the points that are related to the notion of pondered Euler density introduced in this paper.

The complete bare lagrangian reads

$$
\mathcal{L}_{\mathrm{B}}=\mathcal{L}_{g}+\mathcal{L}_{\varphi}
$$

with

$$
\begin{aligned}
\mathcal{L}_{g} & =a_{\mathrm{B}} \mathrm{G}_{6}+b_{\mathrm{B}} R R_{\mu \nu} R^{\mu \nu}+c_{\mathrm{B}} R^{3}+d_{\mathrm{B}} R \square R, \\
\mathcal{L}_{\varphi} & =\frac{1}{2}\left(\nabla_{\mu} \varphi_{\mathrm{B}}\right)^{2}+\frac{1}{2} \xi R \varphi_{\mathrm{B}}^{2}+\frac{\lambda_{\mathrm{B}}}{3 !} \varphi_{\mathrm{B}}^{3}+\frac{1}{2} \eta_{\mathrm{B}} R \varphi_{\mathrm{B}}^{2},
\end{aligned}
$$

where $\xi=(n-2) / 4(n-1)$ and $\eta$ is an independent coupling constant. A flat-space lagrangian of the form (2.9) does not have counterterms linear in $\varphi$, due to the symmetry $\left(\varphi_{1}, \varphi_{2}, \varphi_{3}\right) \rightarrow$ $\left(-\varphi_{1},-\varphi_{2}, \varphi_{3}\right)$.

The trace of the stress-tensor is

$$
\Theta=\frac{1}{\sqrt{g}} \frac{\delta S}{\delta \phi}=\Theta_{g}+\Theta_{\varphi}
$$

Using the formulas reported in the Appendix, we find

$$
\begin{aligned}
\Theta_{g} & =(n-6)\left[a_{\mathrm{B}} \mathrm{G}_{6}+b_{\mathrm{B}} R R_{\mu \nu} R^{\mu \nu}+c_{\mathrm{B}} R^{3}+d_{\mathrm{B}} R \square R\right]-2(n-1) b_{\mathrm{B}} \square\left(R_{\mu \nu} R^{\mu \nu}\right) \\
- & {\left[\frac{n+2}{2} b_{\mathrm{B}}+6(n-1) c_{\mathrm{B}}+\frac{n-2}{2} d_{\mathrm{B}}\right] \square R^{2}-2(n-2) b_{\mathrm{B}} \nabla_{\mu}\left(R^{\mu \nu} \nabla_{\nu} R\right)-4(n-1) d_{\mathrm{B}} \square^{2} R . } \\
\Theta_{\varphi} & =-(n-6) \frac{1}{2} \frac{\lambda_{\mathrm{B}}}{3 !} \varphi_{\mathrm{B}}^{3}-(n-1) \eta_{\mathrm{B}} \square \varphi_{\mathrm{B}}^{2}+\left(\frac{n}{2}-1\right) \mathrm{E}_{\mathrm{B}},
\end{aligned}
$$

where $\mathrm{E}_{\mathrm{B}}=[\mathrm{E}]=\frac{1}{\sqrt{g}} \varphi_{\mathrm{B}} \frac{\delta S_{\varphi}}{\delta \varphi_{\mathrm{B}}}$ is the $\varphi_{\mathrm{B}}$-field equation; $\Theta_{\varphi}$ is the flat-space limit of the trace operator. The coefficient $d$ plays the role of $a^{\prime}$ in four dimensions [3].

An indication in favour of the pondered Euler density is already visible, to all orders in perturbation theory, in the expression of $\Theta$. Indeed, we see that the four total-derivative 
terms of the kind (3.5) are multiplied by three independent coefficients only. This integrability condition must be compatible with the relationships between the coefficients of the pondered Euler density. The crucial test is the ratio between the factors of $\square\left(R_{\mu \nu} R^{\mu \nu}\right)$ and $\nabla_{\mu}\left(R^{\mu \nu} \nabla_{\nu} R\right)$, which equals $5 / 4$ for $n=6$ in $\Theta$. The same ratio appears in the expression (1.7) of $J^{\alpha}$. The remaining factors can be checked only after lengthy work, which we now report on.

\subsection{The composite operator $\varphi^{2}$}

The renormalized field $\varphi$ is defined by

$$
\varphi_{\mathrm{B}}=Z_{1}(\lambda, n) \varphi
$$

In flat space the renormalization of the composite operator $\varphi^{2}$ reads

$$
\left[\varphi^{2}\right]=Z_{2}^{-1}(\lambda, n) \varphi_{\mathrm{B}}^{2}
$$

$Z_{2}$ depends only on $\lambda$, because the other parameters (in particular $\eta$ ) disappear when inserting $\left[\varphi^{2}\right]$ in flat-space correlators. The operator $\square \varphi_{\mathrm{B}}^{2}$ is renormalized in the same way as $\varphi_{\mathrm{B}}^{2}$. We can shift $\eta_{\mathrm{B}} \rightarrow \eta_{\mathrm{B}}+\eta Z_{2}^{-1}, \eta$ being an arbitrary finite parameter, and still obtain finite results. Therefore we write

$$
\eta_{\mathrm{B}}=\left(\eta+L_{\eta}\right) Z_{2}^{-1}, \quad L_{\eta}=\sum_{i=1}^{\infty} \frac{\eta_{i}(\lambda)}{(n-6)^{i}},
$$

where the pole part $L_{\eta}$ does not depend on $\eta$. In curved space the most general expression for $\left[\varphi^{2}\right]$ reads

$$
\begin{aligned}
{\left[\varphi^{2}\right]=} & Z_{2}^{-1} \varphi_{\mathrm{B}}^{2}+2 \mu^{n-6}\left[\left(L_{\kappa^{(1)}}+2 \eta L_{\Lambda^{(1)}}\right) R_{\mu \nu} R^{\mu \nu}\right. \\
& \left.+\left(L_{\kappa^{(2)}}+2 \eta L_{\Lambda^{(2)}}+3 \eta^{2} L_{\Sigma^{(2)}}\right) R^{2}+\left(L_{\kappa^{(3)}}+2 \eta L_{\Lambda^{(3)}}\right) \square R\right]
\end{aligned}
$$

where the pole series $L_{\kappa, \Lambda, \Sigma}$ have the same form as $L_{\eta}$. The $\eta$-dependences follow from the requirement of finiteness of $\left\langle\left[\varphi^{2}\right]\right\rangle$ in curved space. For example, we observe that

$$
\frac{1}{\sqrt{g}} \frac{\delta}{\delta \phi}\left\langle\left[\varphi^{2}\right]\right\rangle=-\left\langle\Theta\left[\varphi^{2}\right]\right\rangle+\frac{1}{\sqrt{g}}\left\langle\frac{\delta}{\delta \phi}\left[\varphi^{2}\right]\right\rangle=\text { (finite) }
$$

We can take the flat-space limit and observe that $\left\langle\Theta\left[\varphi^{2}\right]\right\rangle \mid$ is linear in $\eta$, which implies that the coefficient of the curvature term $\square R$ in $\left[\varphi^{2}\right]$ is linear in $\eta$. The terms $R_{\mu \nu} R^{\mu \nu}$ and $R^{2}$ contribute to the second derivative of $\left\langle\left[\varphi^{2}\right]\right\rangle$ with respect to the conformal factor $\phi$, projected to flat space. Repeating the above argument, we observe that $\left\langle\Theta \Theta\left[\varphi^{2}\right]\right\rangle \mid$ contains the term $\eta^{2}\left\langle\left[\varphi^{2}\right] \square\left[\varphi^{2}\right] \square\left[\varphi^{2}\right]\right\rangle$. This term, however, does not affect $R_{\mu \nu} R^{\mu \nu}$ and therefore the coefficient of $R_{\mu \nu} R^{\mu \nu}$ is linear in $\eta$, while the coefficient $R^{2}$ can be quadratic in $\eta$.

We proceed by observing that the insertion $\frac{\partial S}{\partial \eta}$ must produce finite results in correlators of elementary fields. We have

$$
\frac{\partial S}{\partial \eta}=\frac{\partial S_{g}}{\partial \eta}+\frac{\partial S_{\varphi}}{\partial \eta}
$$




$$
\begin{aligned}
\frac{\partial S_{g}}{\partial \eta} & =\int\left[\frac{\partial a_{\mathrm{B}}}{\partial \eta} \mathrm{G}_{6}+\frac{\partial b_{\mathrm{B}}}{\partial \eta} R R_{\mu \nu} R^{\mu \nu}+\frac{\partial c_{\mathrm{B}}}{\partial \eta} R^{3}+\frac{\partial d_{\mathrm{B}}}{\partial \eta} R \square R\right] \\
\frac{\partial S_{\varphi}}{\partial \eta} & =\int \frac{1}{2} R \varphi_{\mathrm{B}}^{2} Z_{2}^{-1} .
\end{aligned}
$$

We can therefore write, by using (4.2) and equating the various independent pole terms,

$$
\begin{array}{lrl}
a_{\mathrm{B}}=\mu^{n-6}\left(a+L_{a}\right), & b_{\mathrm{B}}=\mu^{n-6}\left(b+L_{b}+\eta L_{\kappa^{(1)}}+\eta^{2} L_{\Lambda^{(1)}}\right), \\
c_{\mathrm{B}}=\mu^{n-6}\left(c+L_{c}+\eta L_{\kappa^{(2)}}+\eta^{2} L_{\Lambda^{(2)}}+\eta^{3} L_{\Sigma^{(2)}}\right), & d_{\mathrm{B}}=\mu^{n-6}\left(d+L_{d}+\eta L_{\kappa^{(3)}}+\eta^{2} L_{\Lambda^{(3)}}\right),
\end{array}
$$

$a, b, c$ and $d$ denoting finite independent parameters, while $L_{a, b, c, d}$ etc. are pole series in $\lambda$ of the same form as $L_{\eta}$.

\subsection{The renormalization-group equations}

Finite and pole parts are related by the condition

$$
\mu \frac{\mathrm{d}}{\mathrm{d} \mu} \mathcal{L}_{\mathrm{B}}=0
$$

In particular, the ambiguities related to the addition of arbitrary finite local terms (3.4) to the action are almost completely fixed. Finite local terms add, unchanged, to the one-particle irreducible effective action $\Gamma$ and affect $\Theta$ via the total derivatives of the list (3.5). This kind of ambiguity does not concern $a_{\mathrm{B}}$, since the $\phi$-variation of $\int \mathrm{G}_{6}$ is zero in six dimensions (see the Appendix). Moreover, these additions have to preserve (4.4) and therefore cannot depend on the renormalized coupling constant $\lambda$. More precisely, if $b_{\mathrm{B}} \rightarrow b_{\mathrm{B}}+b^{\prime} \mu^{n-6}$ with $b^{\prime}$ finite, we have in the limit $n=6, \mu \frac{\mathrm{d} b^{\prime}}{\mathrm{d} \mu}=\beta \frac{\partial b^{\prime}}{\partial \lambda}=0$. The shift $b^{\prime}$ can thus depend on the coupling constant only in families of conformal field theories $(\beta=0)$, such as $\mathrm{N}=4$ supersymmetric Yang-Mills theory. This case, however, does not concern us here. Similar conclusions apply to $c_{\mathrm{B}}$ and $d_{\mathrm{B}}$.

Let us start from $D \eta_{\mathrm{B}}=0$, where

$$
D=\mu \frac{\mathrm{d}}{\mathrm{d} \mu}=\frac{\partial}{\partial \mu}+\hat{\beta}(\lambda) \frac{\partial}{\partial \lambda}+\mu \frac{\mathrm{d} \eta}{\mathrm{d} \mu} \frac{\partial}{\partial \eta}+\cdots
$$

Here $\hat{\beta}=\mu \frac{\mathrm{d} \lambda}{\mathrm{d} \mu}=\frac{1}{2}(n-6) \lambda+\beta$ is the $n$-dimensional beta-function and $\beta=-D \ln Z_{3}\left(\lambda_{\mathrm{B}}=\right.$ $\left.\lambda Z_{3}(\lambda, n) \mu^{-(n-6) / 2}\right)$ is the six-dimensional one. We write

$$
(D+\gamma) Z_{1}^{-1}=0, \quad(D+\delta) Z_{2}^{-1}=0,
$$

$\gamma$ and $\delta$ being finite functions, the anomalous dimensions of $\varphi$ and $\varphi^{2}$, respectively. Calling $\beta_{\eta}(\lambda)$ the finite function $(D-\delta) \eta=\mu \frac{\mathrm{d} \eta}{\mathrm{d} \mu}-\delta \eta$, we can write the renormalization-group equation for the $\eta$-poles in the form

$$
(D-\delta) L_{\eta}=-\beta_{\eta}=\frac{\lambda}{2} \frac{\partial}{\partial \lambda} \eta_{1}(\lambda),
$$

the last equality being obtained by writing the finite term in $(D-\delta) L_{\eta}$ explicitly. 
Similarly, $D a_{\mathrm{B}}=D b_{\mathrm{B}}=D c_{\mathrm{B}}=D d_{\mathrm{B}}=0$, give the other renormalization-group equations. Calling $\beta_{a, b, c, d}(\lambda)$ etc. the various finite parts, functions of the coupling $\lambda$, we have

$$
\begin{aligned}
D a & =\hat{\beta}_{a} \\
D(b, c, d) & =\hat{\beta}_{b, c, d}+\eta \beta_{\kappa^{(1,2,3)}}+\eta^{2} \beta_{\Lambda^{(1,2,3)}}+\eta^{3} \beta_{\Sigma^{(2)}}, \\
\hat{\beta}_{a, b, c, d} & =-(n-6)(a, b, c, d)+\beta_{a, b, c, d}(\lambda), \\
{[(n-6)+D] L_{a} } & =-\beta_{a}(\lambda)=\frac{1}{2 \lambda} \frac{\partial}{\partial \lambda}\left(\lambda^{2} a_{1}\right), \\
{[(n-6)+D] L_{b, c, d}+\beta_{\eta} L_{\kappa^{(1,2,3)}} } & =-\beta_{b, c, d}=\frac{1}{2 \lambda} \frac{\partial}{\partial \lambda}\left(\lambda^{2}(b, c, d)_{1}\right), \\
{[(n-6)+D+\delta] L_{\kappa^{(1,2,3)}}+2 \beta_{\eta} L_{\Lambda^{(1,2,3)}} } & =-\beta_{\kappa^{(1,2,3)}}=\frac{1}{2 \lambda} \frac{\partial}{\partial \lambda}\left(\lambda^{2} \kappa_{1}^{(1,2,3)}\right), \\
{[(n-6)+D+2 \delta] L_{\Lambda^{(1,2,3)}}+3 \beta_{\eta} L_{\Sigma^{(2)}} } & =-\beta_{\Lambda^{(1,2,3)}}=\frac{1}{2 \lambda} \frac{\partial}{\partial \lambda}\left(\lambda^{2} \Lambda_{1}^{(1,2,3)}\right), \\
{[(n-6)+D+3 \delta] L_{\Sigma^{(2)}} } & =-\beta_{\Sigma^{(2)}}=\frac{1}{2 \lambda} \frac{\partial}{\partial \lambda}\left(\lambda^{2} \Sigma_{1}^{(2)}\right) .
\end{aligned}
$$

We have compressed the formulas in a self-evident notation. We recall that $a_{1}, b_{1}$, etc., are the simple poles of the various counterterms.

At a first reading, the reader can follow the various steps focusing on the quantity $a$. The rest is entirely analogous, with the complication due to the renormalization mixing with the operator $\varphi^{2}$.

\subsection{The composite operator $\varphi^{3}$}

The analysis of the composite operator $\varphi^{2}$ has to be repeated for $\varphi^{3}$, by studying the insertions of $\frac{\partial S}{\partial \lambda}$ in correlators. Actually, this procedure produces the integral of the renormalized operator $\left[\varphi^{3}\right]$, rather than $\left[\varphi^{3}\right]$ itself. Therefore $\left[\varphi^{3}\right]$ is obtained up to arbitrary total-derivative terms of the form (3.5) and $\square\left[\varphi^{2}\right]$, multiplied by pole series that we write as

$$
\frac{1}{n-6}\left(f^{(1,2,3,4)}+L_{f^{(1,2,3,4)}}+\eta\left(g^{(1,2,3,4)}+L_{g^{(1,2,3,4)}}\right)+\cdots\right) \quad \text { and } \quad \frac{1}{n-6}\left(e+L_{e}\right),
$$

respectively, $f^{(1,2,3,4)}, g^{(1,2,3,4)}$, etc., and $e$ denoting finite functions. The result for $\frac{1}{3 !} \mu^{-(n-6) / 2}\left[\varphi^{3}\right]$, as well as details of the calculation, are given in the appendix, formula (6.36).

At this point, using (4.1) we obtain the manifestly finite formula for the trace anomaly, which is

$$
\begin{aligned}
\Theta= & -\frac{\hat{\beta}}{3 !}\left[\varphi^{3}\right] \mu^{-(n-6) / 2}+\left(\frac{n}{2}-1+\gamma\right)[\mathrm{E}]-(e+(n-1) \eta) \square\left[\varphi^{2}\right]-\frac{1}{2}\left(\beta_{\eta}+\eta \delta\right) R\left[\varphi^{2}\right] \\
& -\mu^{n-6}\left[\hat{\beta}_{a} \mathrm{G}_{6}+\left(\hat{\beta}_{b, c, d}+\eta \beta_{\kappa^{(1,2,3)}}+\eta^{2} \beta_{\Lambda^{(1,2,3)}}+\eta^{3} \beta_{\Sigma^{(2)}}\right)\left(R R_{\mu \nu} R^{\mu \nu}, R^{3}, R \square R\right)\right] \\
& -\mu^{n-6}\left[\left(f^{(1)}+2(n-1) b+\eta g^{(1)}+\eta^{2} h^{(1)}\right) \square R_{\mu \nu} R^{\mu \nu}\right. \\
& +\left(f^{(2)}+\frac{1}{2}(n+2) b+6(n-1) c+\frac{1}{2}(n-2) d+\eta g^{(2)}+\eta^{2} h^{(2)}\right) \square R^{2} \\
& \left.+\left(f^{(3)}+4(n-1) d+\eta g^{(3)}\right) \square^{2} R+\left(f^{(4)}+2(n-2) b+\eta g^{(4)}+\eta^{2} h^{(4)}\right) \nabla_{\mu}\left(R^{\mu \nu} \nabla_{\nu} R\right)\right]
\end{aligned}
$$


and the consistency relations (6.38) implied by finiteness. Other remarkable relations are obtained by applying the operator $\hat{\beta}^{-1} D \hat{\beta}$ to $\left[\varphi^{3}\right]$. The operator $\hat{\beta}^{-1} D \hat{\beta}$ has the property that it produces finite quantities when acting on finite quantities. Re-expressing $\hat{\beta}\left[\varphi^{3}\right]$ in terms of $\Theta$ and using $D \Theta=0$, finiteness implies the important relations (6.38).

We are now ready to study the lowest-order contributions to some functions. The anomalous dimension $\delta$ of $\left[\varphi^{2}\right]$ is clearly $\mathcal{O}\left(\lambda^{2}\right)$. The quantity $e / \lambda$ is the lowest-order pole of the mixing between $\left[\varphi^{3}\right]$ and $\square\left[\varphi^{2}\right]$, and is $\mathcal{O}\left(\lambda^{3}\right)$. Therefore $e=\mathcal{O}\left(\lambda^{4}\right)$. The first equation of the list (6.38) gives $\beta_{\eta}=\mathcal{O}\left(\lambda^{6}\right)$. Formula (4.5) gives $\eta_{1}(\lambda)=\mathcal{O}\left(\lambda^{6}\right)$ also.

According to the definition $(6.36)$ of $\left[\varphi^{3}\right]$, the quantity $f^{(3)} / \lambda$ is the first pole of the mixing between $\left[\varphi^{3}\right]$ and $\Theta\left(\right.$ via $\left.\square^{2} R\right)$. In particular,

$$
\text { (finite) }=\frac{1}{\sqrt{g}} \frac{\delta}{\delta \phi}\left\langle\left[\varphi^{3}\right]\right\rangle=-\left\langle\Theta\left[\varphi^{3}\right]\right\rangle+\left\langle\frac{1}{\sqrt{g}} \frac{\delta}{\delta \phi}\left[\varphi^{3}\right]\right\rangle .
$$

Projecting this equation to flat space, we see that the pole $f^{(3)} / \lambda$ is cancelled by the first pole of $\left\langle\Theta\left[\varphi^{3}\right]\right\rangle$. This is at least $\mathcal{O}\left(\lambda^{3}\right)$, as we now show, so that we expect $f^{(3)}=\mathcal{O}\left(\lambda^{4}\right)$.

In flat space, the operator $\Theta(x)$ reads

$$
\Theta \mid=-\frac{\hat{\beta}}{3 !}\left[\varphi^{3}\right] \mu^{-(n-6) / 2}+\left(\frac{n}{2}-1+\gamma\right)[\mathrm{E}]-(e+(n-1) \eta) \square\left[\varphi^{2}\right] .
$$

We recall that $\beta=\mathcal{O}\left(\lambda^{3}\right)$, while $e=\mathcal{O}\left(\lambda^{4}\right)$. Moreover, we can write $\hat{\beta}=\frac{1}{2}(n-6) \lambda+\beta=\mathcal{O}\left(\lambda^{3}\right)$. Indeed, the term proportional to $(n-6)$ selects a higher-loop singularity, and we can formally say that $(n-6)$ is $\mathcal{O}\left(\lambda^{2}\right)$. Finally, $\gamma=\mathcal{O}\left(\lambda^{2}\right)$, but we can neglect the term in $[\mathrm{E}]$ in $\left\langle\widetilde{\Theta} \mid\left[\varphi^{3}\right]\right\rangle$, since a simple functional argument shows that $\langle[\mathrm{E}] P\rangle=0$ for any operator $P$. We have thus proved that $f^{(3)}=\mathcal{O}\left(\lambda^{4}\right)$. There is, however, a cancellation between the two-loop contribution singled out by the term $\frac{\lambda}{2}(n-6)$ in $\hat{\beta}$ and the one-loop contribution coming from $\beta$. This enhancement, which produces the result that $f^{(3)}$ is actually $\mathcal{O}\left(\lambda^{6}\right)$, will be proved in the next section. Precisely, it will be proved that $\beta_{d}=\mathcal{O}\left(\lambda^{8}\right)$. Then the claimed result follows from the fifth formula from the bottom of the list 6.38).

We now consider the quantity $g^{(3)} / \lambda$. We can repeat the above analysis and consider the $\eta$-term in $\Theta \mid$. The pole $g^{(3)} / \lambda$ cancels the first pole of $\left\langle\left[\varphi^{3}\right] \square[\varphi]^{2}\right\rangle$, which is $\mathcal{O}(\lambda)$. So, $g^{(3)}=\mathcal{O}\left(\lambda^{2}\right)$. No enhancement takes place here.

Moreover, the fourth formula of (6.38) from the bottom implies that $\beta_{\kappa^{(3)}}=\mathcal{O}\left(\lambda^{4}\right)$. The same result can be proved, using formula (4.3) projected to flat space and the definition (4.2) of $\left[\varphi^{2}\right]: \kappa_{1}^{(3)}$ is the first-loop pole of $\left\langle\beta\left[\varphi^{3}\right]\left[\varphi^{2}\right]\right\rangle$. Taking the $\eta$-term, a similar argument proves that $\beta_{\Lambda^{(3)}}=\mathcal{O}(1)$. Similar considerations show that $f^{(1,2,4)}=\mathcal{O}\left(\lambda^{6}\right), g^{(1,2,4)}=\mathcal{O}\left(\lambda^{2}\right), \beta_{\kappa^{(1,2)}}=$ $\mathcal{O}\left(\lambda^{4}\right), \beta_{\Lambda^{(1,2)}}=\mathcal{O}(1)$.

Before closing this section, let us discuss the parameter $\eta$ in detail, in particular the renormalization-group equation $D \eta=\delta \eta+\beta_{\eta}$. Writing, $\eta=u(\lambda) \eta^{\prime}+v(\lambda)$, we have $D \eta^{\prime}=0$, and for $n=6, u(\lambda) \sim \lambda^{\delta_{1} / \beta_{1}}$ (where $\delta=\delta_{1} \lambda^{2}+\mathcal{O}\left(\lambda^{4}\right), \beta=\beta_{1} \lambda^{3}+\mathcal{O}\left(\lambda^{5}\right)$ ) and $v(\lambda)=\mathcal{O}\left(\lambda^{4}\right)$. The parameter $\eta^{\prime}$ is the true independent coupling constant of the term $R \varphi^{2}$ in the lagrangian and can be set to zero. A non-zero value of this parameter would produce non-integer powers 
of $\lambda$ and therefore the choice $\eta^{\prime}=0$ is universal. This can be better apppreciated by observing that the solution for $u(\lambda)$ reads

$$
u(\lambda)=\exp \left(\int_{\lambda_{0}}^{\lambda} \frac{\delta\left(\lambda^{\prime}\right)}{\beta\left(\lambda^{\prime}\right)} \mathrm{d} \lambda^{\prime}\right) .
$$

The arbitrariness of $\lambda_{0}$ is the same as the arbitrariness of $\eta^{\prime}$. Now, $u(\lambda)$ is not proportional to the beta function, so $\Theta$ contains a term $\eta^{\prime} u(\lambda) \square\left[\varphi^{2}\right]$. To restore scale invariance at the fixed points, $\Theta$ should be proportional to the beta function, up to field equations. Therefore we have to set $\eta^{\prime}=0$. The remaining $\square\left[\varphi^{2}\right]$-term is indeed proportional to the beta function, since using the first relation of (6.38) the coefficient of the $\square\left[\varphi^{2}\right]$-term in $\Theta$ reads

$$
e+(n-1) \eta=(n-1) \frac{\hat{\beta}}{\delta} \frac{\partial \eta}{\partial \lambda} .
$$

Observe that $\frac{1}{\delta} \frac{\partial \eta}{\partial \lambda}$ is regular also in the free-field limit. For a generic interacting fixed point, $\delta_{*}>0$. We conclude that only the choice $\eta^{\prime}=0$ is consistent with the RG interpolation problem. At a criticality we have, correctly,

$$
\Theta \mid=\left(\frac{n}{2}-1+\gamma_{*}\right)[\mathrm{E}],
$$

$\gamma_{*}$ being the anomalous dimension of the elementary field $\varphi$.

\subsection{The $\Theta$ two-, three- and four-point function}

We now explain the basic reason why the pondered Euler density comes out naturally of the calculation. It is convenient to define

$$
\widetilde{\Theta}=\sqrt{g} \Theta=\frac{\delta S}{\delta \phi}
$$

We study the first, second and third derivatives of $\tilde{\Theta}$, which are related to the two-, threeand fourth-point functions of $\widetilde{\Theta}$ itself. The derivatives of a finite quantity, like $\langle\tilde{\Theta}\rangle$, are finite. Therefore we have, for example,

$$
\frac{\delta\langle\widetilde{\Theta}\rangle}{\delta \phi}=-\langle\widetilde{\Theta} \widetilde{\Theta}\rangle+\left\langle\frac{\delta}{\delta \phi} \tilde{\Theta}\right\rangle=(\text { finite })
$$

First, we can work out relations between the poles. Then, using the RG equations, we can obtain information about the finite parts.

The two-point function fixes the order of the first radiative correction to the coefficient $d$, in particular the coefficient $d_{1}$ of the first-pole term.

Let us define

$$
\overline{\widetilde{\Theta}}=\widetilde{\Theta}-\left(\frac{n}{2}-1\right) \sqrt{g} \mathrm{E}=\widetilde{\Theta}-\left(\frac{n}{2}-1\right) \tilde{\mathrm{E}},
$$


so that equation 4.9 can be re-expressed as

$$
-\langle\overline{\widetilde{\Theta}} \overline{\widetilde{\Theta}}\rangle+\left\langle\frac{\delta}{\delta \phi} \tilde{\Theta}\right\rangle=(\text { finite }) .
$$

We can neglect the term in $[\mathrm{E}]$ in $\langle\widetilde{\Theta} \tilde{\Theta}\rangle$, since $\langle[\mathrm{E}] P\rangle=0$ for any $P$. We have

$$
\overline{\widetilde{\Theta}} \mid=\frac{n-6}{2} \frac{\lambda_{\mathrm{B}}}{3 !} \varphi_{\mathrm{B}}^{3}-(n-1) \eta_{\mathrm{B}} \square \varphi_{\mathrm{B}}^{2}=-\frac{\hat{\beta}}{3 !}\left[\varphi^{3}\right] \mu^{-(n-6) / 2}+\gamma[\mathrm{E}]-(e+(n-1) \eta) \square\left[\varphi^{2}\right] .
$$

We can write in general

$$
\overline{\widetilde{\Theta}} \mid=\hat{\beta} Q, \quad Q=-\frac{1}{3 !}\left[\varphi^{3}\right] \mu^{-(n-6) / 2}+\frac{\gamma}{\hat{\beta}}[\mathrm{E}]-(n-1) \frac{1}{\delta} \frac{\partial \eta}{\partial \lambda} \square\left[\varphi^{2}\right] .
$$

The operator $Q$ is particularly useful. It contains poles, but we will see that they are harmless, since they multiply just the field equations. An important property of $Q$ is $D(\hat{\beta} Q)=0$ in flat space.

We recall that $\beta, \hat{\beta}=\mathcal{O}\left(\lambda^{3}\right)$, while $e, \eta=\mathcal{O}\left(\lambda^{4}\right), \gamma=\mathcal{O}\left(\lambda^{2}\right)$. Naively, we expect $d_{1}=\mathcal{O}\left(\lambda^{6}\right)$. We now prove that there is an enhancement due to the RG equations and $d_{1}=\mathcal{O}\left(\lambda^{8}\right)$. This phenomenon is similar to a phenomenon occurring in four dimensions [17, but here is implies that the first radiative corrections to the quantity $a$ are four-loop, rather than three-loop. We write the poles of the two-point function as

$$
\langle Q(x) Q(0)\rangle=\mu^{n-6}\left(\frac{n-6}{\hat{\beta}}\right)^{2} L_{X} \square^{3} \delta(x)+\text { (finite) }
$$

where $L_{X}$ is a pole series of the usual form. The divergent terms are local, since subdivergences are originated only from vertex graphs of elementary fields and at most one insertion of $Q$. Therefore they are automatically subtracted away.

We see that the first pole $X_{1}$ is, by definition, $\mathcal{O}\left(\lambda^{2}\right)$. Writing $X_{1}=x_{1} \lambda^{2}$ we find by explicit computation

$$
x_{1}=-\frac{1}{4 \cdot 6 !} \frac{1}{(4 \pi)^{6}}
$$

Formula 4.10) gives

$$
\widetilde{d}_{1}=\frac{X_{3}}{200}
$$

where $X_{3}$ is the triple pole of $L_{X}$. We put a tilde on $d_{1}$ to stress that the first pole in consideration does not come just from $L_{d}$, but from the full sum $L_{d}+\eta L_{\kappa^{(3)}}+\eta^{2} L_{\Lambda^{(3)}}$ appearing in the definition of $d_{\mathrm{B}}$ given in section 4.1. The $\eta$-contributions are indeed $\mathcal{O}\left(\lambda^{8}\right)$ also.

We can compute $X_{3}$ as follows. We apply $\hat{\beta}^{-2} D \hat{\beta}^{2}$ to (4.11) and observe that $\hat{\beta}^{-2} D \hat{\beta}^{2}$ (finite) = (finite). Moreover, we recall that $D(\hat{\beta} Q)=0$. We have, therefore:

$$
\left(\frac{n-6}{\hat{\beta}}\right)^{2}[(n-6)+D] L_{X}=(\text { finite })=\frac{2}{\lambda^{3}} \frac{\partial}{\partial \lambda}\left(\lambda^{2} X_{1}\right) .
$$


The right-hand side is obtained by writing the unique finite contribution to the left-hand side. Solving the recursion relations we arrive at

$$
\frac{\partial}{\partial \lambda}\left(\lambda^{2} X_{3}\right)=-8 \frac{\beta}{\lambda^{2}} \int^{\lambda} \mathrm{d} \lambda^{\prime} \lambda^{\prime 4} X_{1}\left(\lambda^{\prime}\right) \frac{\partial}{\partial \lambda^{\prime}}\left(\frac{\beta\left(\lambda^{\prime}\right)}{\lambda^{\prime 3}}\right)
$$

and therefore

$$
X_{3}=-\frac{1}{5} \beta_{1} \beta_{2} x_{1} \lambda^{8}, \quad \widetilde{d}_{1}=\frac{1}{4000 \cdot 6 !} \beta_{1} \beta_{2} \frac{\lambda^{8}}{(4 \pi)^{6}} .
$$

We now study the second derivative of $\langle\widetilde{\Theta}(x)\rangle$ with respect to $\phi$,

$$
\langle\widetilde{\Theta}(x) \widetilde{\Theta}(y) \widetilde{\Theta}(z)\rangle-\left\langle\frac{\delta \widetilde{\Theta}(x)}{\delta \phi(y)} \widetilde{\Theta}(z)\right\rangle-\left\langle\frac{\delta \widetilde{\Theta}(x)}{\delta \phi(z)} \widetilde{\Theta}(y)\right\rangle-\left\langle\frac{\delta \widetilde{\Theta}(y)}{\delta \phi(z)} \widetilde{\Theta}(x)\right\rangle+\left\langle\frac{\delta^{2} \widetilde{\Theta}(x)}{\delta \phi(y) \delta \phi(z)}\right\rangle=\text { (finite). }
$$

The $\tilde{\Theta}$ three-point function contains non-local divergences associated with vertices with two $\tilde{\Theta}$-legs in the same point. These, however, are subtracted away by the middle terms of (4.14) and the remaining overall divergence (the last term on the left-hand side of (4.14)) is indeed purely local. (4.14) can be written in the schematic form

$$
\langle\overline{\widetilde{\Theta}} \overline{\widetilde{\Theta}} \overline{\widetilde{\Theta}}\rangle-3\left\langle\overline{\widetilde{\Theta}} \frac{\Delta \overline{\widetilde{\Theta}}}{\Delta \phi}\right\rangle+\left\langle\frac{\delta^{2} \tilde{\Theta}(x)}{\delta \phi^{2}}\right\rangle=(\text { finite }),
$$

where we have defined

$$
\frac{\Delta \overline{\widetilde{\Theta}}}{\Delta \phi}=\frac{\delta \overline{\widetilde{\Theta}}}{\delta \phi}-\frac{n-2}{2} \varphi_{\mathrm{B}} \frac{\delta \overline{\widetilde{\Theta}}}{\delta \varphi_{\mathrm{B}}}
$$

and used

$$
\langle[\tilde{\mathrm{E}}] A B\rangle=\left\langle\varphi_{\mathrm{B}} \frac{\delta A}{\delta \varphi_{\mathrm{B}}} B\right\rangle+\left\langle A \varphi_{\mathrm{B}} \frac{\delta B}{\delta \varphi_{\mathrm{B}}}\right\rangle
$$

We write

$$
\frac{\Delta \overline{\widetilde{\Theta}}(x)}{\Delta \phi(y)} \mid=\hat{\beta} \frac{\Delta Q(x)}{\Delta \phi(y)}+8(n-1)^{2} d_{\mathrm{B}} \square^{3} \delta(x-y) .
$$

The $d_{\mathrm{B}}$-term here originates disconnected contributions to the correlators. There is no such contribution in (4.15), but there will be some in the four-point function. Moreover, we write

$$
\langle Q Q Q\rangle_{*}-\frac{3}{\hat{\beta}}\left\langle Q \frac{\Delta Q}{\Delta \phi}\right\rangle_{*}=\mu^{n-6}\left(\frac{n-6}{\hat{\beta}}\right)^{3} L_{Y} \times \text { local structure }+ \text { (finite) } .
$$

Here there are also non-local divergent terms coming from subdivergences with a vertex of two $Q$-legs in the same point. Not all of them are automatically subtracted by the second term of (4.17). The star subscript means that we subtract them away by hand.

The local structure of formula (4.17) is a sum of expressions of the form $\partial^{p} \delta(x-y) \partial^{q} \delta(x-z)$, with $p+q=6, p, q \geq 2$, fixed by the term $\left\langle\frac{\delta^{2} \tilde{\Theta}(x)}{\delta \phi^{2}}\right\rangle \mid$ in (4.15).

Applying $\hat{\beta}^{-3} D \hat{\beta}^{3}$ and using $D \frac{\Delta \widetilde{\widetilde{\Theta}}}{\Delta \phi}=0$ we can prove an RG equation similar to 4.12) and show that there is an enhancement again. This would be straightforward in the absence of the 
subtractions just mentioned. The result is that one finds an equation of the type (4.12), but with a non-zero right-hand side. It is easy to see, however, by direct inspection of the structure and graphs of (4.17), that these corrections are of higher order $\left(\mathcal{O}\left(\lambda^{4}\right)\right.$ for $Y_{1}, \mathcal{O}\left(\lambda^{6}\right)$ for $Y_{2}$, etc. up to $\mathcal{O}\left(\lambda^{10}\right)$ for $\left.Y_{4}\right)$ and we do not need any enhancement to discard these. This fact is actually natural, since subdivergences cannot contribute to the lowest orders, which is all what we are interested in here. In four dimensions the matter is entirely similar [17, 18]. We also remark, without giving further details, that there is one peculiar local structure which is not affected by non-local subdivergences. This is not sufficient, however, for us, since we need two conditions. For this reason we are compelled to inspect the subdivergences originated by the vertices with two $Q$-legs in the same point. The matter is simpler for the four-point function, where there is one special local structure and we need precisely one condition.

A direct inspection of (4.17) proves that to the lowest order $Y_{1}=\mathcal{O}\left(\lambda^{2}\right)$, so that the RG equations imply $Y_{2}=\mathcal{O}\left(\lambda^{4}\right)$ and $Y_{3}=\mathcal{O}\left(\lambda^{6}\right)$ and one naively expects $Y_{4}=\mathcal{O}\left(\lambda^{8}\right)$. The enhancement fixes instead $Y_{4}=\mathcal{O}\left(\lambda^{10}\right)$.

To help studying $Y_{1}$ we observe that

$$
\frac{\Delta Q}{\Delta \phi}=\frac{1}{2}(6-n) Q+\eta \text { terms. }
$$

The $\eta$-terms are of order $\mathcal{O}(\lambda)$, at least, quadratic in $\varphi$, and also contain poles. The important fact is that $\Delta Q / \Delta \phi$ is of higher order with respect to $Q$. Finally, in $Q$ the field equation $\mathrm{E}$ is multiplied by $\gamma / \hat{\beta}$, which is formally $\mathcal{O}(\lambda /(n-6))$. Let us note that $\langle[\mathrm{E}][\mathrm{E}][\mathrm{E}]\rangle=0$, which follows from (4.16).

Now, $Y_{1}=\mathcal{O}\left(\lambda^{2}\right)$ means a simple pole in (4.17) of order $1 / \lambda$. This can be originated, indeed, by the denominator $1 / \hat{\beta}$, but it is easy to check that contributions worse tan this (finite or divergent, local or non-local) simply do not exist in (4.17).

We conclude the analysis of the three-point function by stating that

$$
\left\langle\frac{\delta^{2} \widetilde{\Theta}(x)}{\delta \phi(y) \delta \phi(z)}\right\rangle \mid=(\text { finite })
$$

to the order $\lambda^{8}$ included. In particular, we study the simple poles of (4.18) and set them to zero. These are linear combinations of $b_{1}, c_{1}$ and $d_{1}$ (see below). Because of the one-to-one correspondence between pole terms and finite terms, it is like setting the finite part of $\frac{\delta^{2} \widetilde{\Theta}(x)}{\delta \phi(y) \delta \phi(z)} \mid$ to zero and this is the first step for the definition of the pondered Euler density.

Then, the argument is repeated for the four-point function, first writing

$$
-\langle\overline{\widetilde{\Theta}} \overline{\widetilde{\Theta}} \overline{\widetilde{\Theta}} \overline{\widetilde{\Theta}}\rangle-3\left\langle\frac{\Delta \overline{\widetilde{\Theta}}}{\Delta \phi} \frac{\Delta \overline{\widetilde{\Theta}}}{\Delta \phi}\right\rangle-4\left\langle\overline{\widetilde{\Theta}} \frac{\Delta^{2} \overline{\widetilde{\Theta}}}{\Delta \phi^{2}}\right\rangle+6\left\langle\overline{\widetilde{\Theta}} \overline{\widetilde{\Theta}} \frac{\Delta \widetilde{\widetilde{\Theta}}}{\Delta \phi}\right\rangle+\left\langle\frac{\delta^{3} \widetilde{\Theta}(x)}{\delta \phi^{3}}\right\rangle=\text { (finite), }
$$

and then observing that this formula reduces to

$$
\left\langle\frac{\delta^{3} \widetilde{\Theta}(x)}{\delta \phi(y) \delta \phi(z) \delta \phi(w)}\right\rangle \mid=(\text { finite })
$$


to the order we are interested in. This result can be obtained following the same procedure that we have applied for the two- and three-point functions and keeping into account that, despite there are disconnected contributions, they sum up to finite terms and can therefore be neglected throughout. Indeed, the disconnected contributions to (4.19) read in flat space

$$
-3\langle\overline{\widetilde{\Theta}} \overline{\widetilde{\Theta}}\rangle\langle\overline{\widetilde{\Theta}} \overline{\widetilde{\Theta}}\rangle-3\left\langle\frac{\Delta \overline{\widetilde{\Theta}}}{\Delta \phi}\right\rangle\left\langle\frac{\Delta \overline{\widetilde{\Theta}}}{\Delta \phi}\right\rangle+6\langle\overline{\widetilde{\Theta}} \overline{\widetilde{\Theta}}\rangle\left\langle\frac{\Delta \overline{\widetilde{\Theta}}}{\Delta \phi}\right\rangle=-3\left(\langle\overline{\widetilde{\Theta}} \overline{\widetilde{\Theta}}\rangle-\left\langle\frac{\Delta \overline{\widetilde{\Theta}}}{\Delta \phi}\right\rangle\right)^{2}=\text { (finite), }
$$

using (4.10).

We study the order- $\lambda^{8}$ simple poles of (4.18) and (4.20). The relevant contribution to $\widetilde{\Theta}$ is

$$
\frac{1}{n-6}\left[a_{2} \tilde{\mathrm{G}}_{6}-10 \widetilde{b}_{1} \square\left(R_{\mu \nu} R^{\mu \nu}\right)-8 \widetilde{b}_{1} \nabla_{\mu}\left(R^{\mu \nu} \nabla_{\nu} R\right)-\left(30 \widetilde{c}_{1}+4 \widetilde{b}_{1}+2 \widetilde{d}_{1}\right) \square R^{2}-20 \widetilde{d}_{1} \square^{2} R\right] .
$$

We recall that the tildes on $b_{1}, c_{1}, d_{1}$ denote the full first poles of $b_{\mathrm{B}}, c_{\mathrm{B}}, d_{\mathrm{B}}$ (see section 4.1). Condition (4.18) gives

$$
\widetilde{b}_{1}=5 \widetilde{d}_{1}, \quad \widetilde{c}_{1}=-\frac{39}{30} \widetilde{d}_{1}
$$

while 4.20 ) imposes

$$
a_{2}=\frac{25}{6} \widetilde{d}_{1}
$$

so that (4.21) reads

$$
\frac{25}{6(n-6)} \tilde{d}_{1} \tilde{\mathrm{G}}_{6}
$$

This is the form of the simple poles. We now show that the finite parts (as well as the other poles) are related in a similar way. Formula (4.23) is the analogue of the identification $a=a^{\prime}$ of ref. [3].

We compare the radiative correction to the coefficient $-\beta_{a}$ of $\mathrm{G}_{6}$ with the prediction (2.10). We recall that

$$
-\beta_{a}=\frac{1}{2 \lambda} \frac{\partial}{\partial \lambda}\left(\lambda^{2} a_{1}\right)
$$

according to (4.6). The same renormalization-group equations (4.6) give

$$
-\beta \frac{\partial a_{1}}{\partial \lambda}=\frac{1}{2 \lambda} \frac{\partial}{\partial \lambda}\left(\lambda^{2} a_{2}\right) .
$$

Here, $a_{2}=\frac{25}{6} \tilde{d}_{1}$ can be read from (4.13). The additive constant in $a_{1}$ is not fixed by our procedure. It is the free-field value of the anomaly, which can however be computed directly 20]. Combining the above results we have

$$
\Theta=\left(\text { const. }+\frac{41}{7464960} \frac{\lambda^{6}}{(4 \pi)^{12}}\right) \mathrm{G}_{6}+\text { rest }
$$

to the fourth-loop order included, as we wished to show.

Recalling the procedure with which $\tilde{d}_{1}$ was calculated, this result shows the direct relationship between the coefficient of the Euler density (which contributes to the $\Theta$ four-point function, 
since it is cubic in the Riemann tensor) and the $\Theta$ two-point function, in agreement with the ideas of [3]. This relationship in non-trivial and would not be naively expected to hold. Only when analysing the induced effective action for the conformal factor does one uncover that a very simple positivity property is actually equivalent to the claimed relationship to all-orders in perturbation theory [3]. We stress that the positivity arguments of [3] do apply here and imply that the induced action for the conformal factor is negative definite throughout the RG flow.

We now comment on the remaining terms of (4.26). The terms of the list (3.4) should be proportional to the beta function, by the integrability condition, while the terms of the list (3.5) should reconstruct $\tilde{\mathrm{G}}_{6}$, as in (4.24). However, this is not straightforward, due to the renormalization mixing betwen $\Theta$ and $\square\left[\varphi^{2}\right]$. Having already obtained the desired result, namely (4.26), we will not pursue this matter further. Nevertheless, we conclude this section by showing that, consistently with what we have just said, there would be no trouble in the absence of the $\square\left[\varphi^{2}\right]$-mixing. In this case, the equations reported in the appendix (as well as our discussion so far) would simplify enormously: $\eta, e, \beta_{\eta}, \kappa^{(1,2,3)}, \Lambda^{(1,2,3)}, \Xi^{(2)}, g^{(1,2,3,4)}, h^{(1,2,4)} \rightarrow 0$. We are left with $a, b, c, d$ and $f^{(1,2,3,4)}$, related via an oversimplified version of (6.38) and (4.6), namely — for $n=6$ -

$$
\begin{aligned}
5 \beta_{b} & =\frac{\beta}{\lambda} f^{(1)}, \quad 2 \beta_{b}+15 \beta_{c}+\beta_{d}=\frac{\beta}{\lambda} f^{(2)}, \\
10 \beta_{d} & =\frac{\beta}{\lambda} f^{(3)}, \quad 4 \beta_{b}=\frac{\beta}{\lambda} f^{(4)}, \quad D(b, c, d)=\beta \frac{\partial(b, c, d)}{\partial \lambda}=\beta_{b, c, d} .
\end{aligned}
$$

We see that $\beta_{b, c, d}$, coefficients of the terms (3.4) in $\Theta$, are proportional to the beta function, in agreement with the integrability condition. The coefficients of the terms (3.5),

$-\left(f^{(1)}+10 b\right) \square R_{\mu \nu} R^{\mu \nu}-\left(f^{(2)}+4 b+30 c+2 d\right) \square R^{2}-\left(f^{(3)}+20 d\right) \square^{2} R-\left(f^{(4)}+8 b\right) \nabla_{\mu}\left(R^{\mu \nu} \nabla_{\nu} R\right)$,

can be found by repeating the above procedure for $\beta_{a}$. We have, using (4.27),

$$
-\frac{1}{2 \lambda} \frac{\partial}{\partial \lambda}\left\{\lambda^{2}\left[10 b \square R_{\mu \nu} R^{\mu \nu}+(4 b+30 c+2 d) \square R^{2}+20 d \square^{2} R+8 b \nabla_{\mu}\left(R^{\mu \nu} \nabla_{\nu} R\right)\right]\right\} .
$$

Now, (4.6) gives also equations that are analogous to (4.25):

$$
-\beta \frac{\partial b}{\partial \lambda}=\frac{1}{2 \lambda} \frac{\partial}{\partial \lambda}\left(\lambda^{2} b_{1}\right), \quad-\beta \frac{\partial c}{\partial \lambda}=\frac{1}{2 \lambda} \frac{\partial}{\partial \lambda}\left(\lambda^{2} c_{1}\right), \quad-\beta \frac{\partial d}{\partial \lambda}=\frac{1}{2 \lambda} \frac{\partial}{\partial \lambda}\left(\lambda^{2} d_{1}\right) .
$$

Therefore, the relations (4.22) and (4.23) between $a_{2}$ and $b_{1}, c_{1}, d_{1}$ are promoted to similar relations between $a_{1}$ and $b, c, d$ (up to the usual additive constants), so that, in turn, (4.26) is promoted to

$$
\Theta=\frac{1}{2 \lambda} \frac{\partial}{\partial \lambda}\left(\lambda^{2} a_{1}\right) \tilde{\mathrm{G}}_{6}+\text { rest. }
$$

\section{General dimension}

We now discuss the case of generic $n$. The pondered Euler density has the form

$$
\tilde{\mathrm{G}}_{n}=\mathrm{G}_{n}+\nabla_{\alpha} J_{n}^{\alpha}=\mathrm{G}_{n}+\cdots+p_{n} \square^{n / 2-1} R, \quad J_{n}^{\alpha}=\cdots+p_{n} \nabla^{\alpha} \square^{\frac{n}{2}-2} R .
$$


The dots stand for a list of trivial total derivative terms, which are at least quadratic in the curvature tensor. The weights of the various terms in the list are chosen in such a way that on conformally-flat metrics

$$
\sqrt{g} \tilde{\mathrm{G}}_{n}=-2(n-1) p_{n} \square^{\frac{n}{2}} \phi .
$$

Only the coefficient $p_{n}$ in $(5.28)$ is relevant to quantum irreversibility and the definition of $\tilde{\mathrm{G}}_{n}$ makes it easily calculable. We plan to devote a separate paper to the general mathematical constrution of $\tilde{\mathrm{G}}_{n}$.

The computation of $p_{n}$ proceeds as follows. The Euler characteristic of an $n$-dimensional sphere $S^{n}$, equal to 2 , can be written as

$2=\frac{(-1)^{\frac{n}{2}}}{2^{\frac{3 n}{2}} \pi^{\frac{n}{2}}\left(\frac{n}{2}\right) !} \int_{S^{n}} \sqrt{g} \mathrm{G}_{n} \mathrm{~d}^{n} x=\frac{(-1)^{\frac{n}{2}}}{2^{\frac{3 n}{2}} \pi^{\frac{n}{2}}\left(\frac{n}{2}\right) !} \int_{S^{n}} \sqrt{g} \tilde{\mathrm{G}}_{n} \mathrm{~d}^{n} x=-\frac{2(-1)^{\frac{n}{2}}(n-1)}{2^{\frac{3 n}{2}} \pi^{\frac{n}{2}}\left(\frac{n}{2}\right) !} p_{n} \int_{S^{n}} \square^{\frac{n}{2}} \phi \mathrm{d}^{n} x$,

where

$$
\mathrm{G}_{n}=(-1)^{\frac{n}{2}} \varepsilon_{\mu_{1} \nu_{1} \cdots \mu_{\frac{n}{2}} \nu_{\frac{n}{2}}} \varepsilon^{\alpha_{1} \beta_{1} \cdots \alpha_{\frac{n}{2}} \beta_{\frac{n}{2}}} \prod_{i=1}^{\frac{n}{2}} R_{\alpha_{i} \beta_{i}}^{\mu_{i} \nu_{i}}
$$

A sphere has the metric $\mathrm{d} s^{2}=\frac{\mathrm{d} x^{2}}{\left(1+x^{2}\right)^{2}}$, so that $\phi=-\ln \left(1+x^{2}\right)$. A tedious, but straightforward, computation gives

$$
\square^{\frac{n}{2}} \phi=2^{\frac{3 n}{2}-1}(-1)^{\frac{n}{2}} \Gamma\left(\frac{n}{2}\right)(n-1) ! ! \frac{1}{\left(1+|x|^{2}\right)^{n}}
$$

and

$$
\int \square^{\frac{n}{2}} \phi=2^{n}(-1)^{\frac{n}{2}} \pi^{\frac{n}{2}} \Gamma\left(\frac{n}{2}\right)
$$

We have therefore the result

$$
p_{n}=-\frac{2^{\frac{n}{2}} n}{2(n-1)},
$$

which agrees with the known values in $n=4(1.2)$ and $n=6$ (1.7). With the help of a computer we have checked this formula for $n=8$, where the pondered Euler density reads

$$
\tilde{\mathrm{G}}_{8}=\mathrm{G}_{8}-\frac{64}{7} \square^{3} R+\nabla_{\alpha} J_{8 \text { red }}^{\alpha},
$$

with

$$
\begin{aligned}
J_{8 \text { red }}^{\alpha}= & \frac{1984}{147}\left(\nabla^{\alpha} \square R\right) R-\frac{1280}{21}\left(\nabla_{\mu} \square R\right) R^{\mu \alpha}+\frac{1024}{63}\left(\nabla_{\rho} R^{\alpha \mu}\right) R_{\mu \nu} R^{\nu \rho}+\frac{21376}{441}\left(\nabla^{\alpha} R_{\mu \nu}\right) R^{\mu \nu} R \\
& -\frac{256}{3}\left(\nabla_{\mu} R_{\rho \sigma}\right) R^{\rho \sigma} R^{\mu \alpha}-\frac{9728}{1029}\left(\nabla^{\alpha} R\right) R^{2}+\frac{74752}{3087}\left(\nabla_{\mu} R\right) R R^{\mu \alpha} \\
& -\frac{4800}{7}\left(\nabla^{\alpha} R^{\mu \nu}\right) \nabla_{\mu} \nabla_{\nu} R-\frac{256}{3}\left(\nabla^{\mu} R^{\nu \rho}\right) \nabla^{\alpha} \nabla_{\rho} R_{\mu \nu}+\frac{64}{7}\left(\nabla_{\mu} R\right) \square R^{\mu \alpha} \\
& +\frac{2752}{3}\left(\nabla^{\alpha} R_{\mu \nu}\right) \square R^{\mu \nu}+\frac{101120}{147}\left(\nabla^{\nu} R^{\mu \alpha}\right) \nabla_{\mu} \nabla_{\nu} R-\frac{23552}{21}\left(\nabla^{\nu} R^{\mu \alpha}\right) \square R_{\mu \nu .} .
\end{aligned}
$$

On conformally-flat metrics we have

$$
\sqrt{g} \tilde{\mathrm{G}}_{n}=2^{\frac{n}{2}} n \square{ }^{\frac{n}{2}} \phi
$$


for $n \geq 2$. For $n=2, \mathrm{G}_{2}=\tilde{\mathrm{G}}_{2}$.

We are ready to write the general formula for the $a$-flow. We normalize $a$ in such a way that the trace anomaly equation reads at criticality

$$
\Theta=a_{n} \tilde{\mathrm{G}}_{n}+\text { conformal invariants }=2^{\frac{n}{2}} n a_{n} \mathrm{e}^{-n \phi} \square^{\frac{n}{2}} \phi .
$$

The two-point function reads

$$
\langle\Theta(x) \Theta(y)\rangle=-2^{\frac{n}{2}} n a_{n} \square^{\frac{n}{2}} \delta(x-y)
$$

and the expression for the $a$-flow is:

$$
a_{n}^{\mathrm{UV}}-a_{n}^{\mathrm{IR}}=\frac{1}{2^{\frac{3 n}{2}-1} n \Gamma(n+1)} \int \mathrm{d}^{n} x|x|^{n}\langle\Theta(x) \Theta(0)\rangle,
$$

using $\square^{\frac{n}{2}}|x|^{n}=n(2 n-2) !$ !.

Using [20], we can read the values of $a_{n}$ for free fields in arbitrary dimension and normalize $a_{n}$ so that it equals 1 for a real scalar field, and reads in general

$$
N_{s}+f_{n} N_{f}+v_{n} N_{v}
$$

for free-field theories.

The odd-dimensional formula of quantum irreversibility that we propose is obtained by putting $n=$ odd in (5.30). For example,

$$
a_{\mathrm{UV}}-a_{\mathrm{IR}}=\frac{1}{144 \sqrt{2}} \int \mathrm{d}^{3} x|x|^{3}\langle\Theta(x) \Theta(0)\rangle
$$

in three dimensions. The normalization (5.31), as well as the free-field values of $a$, can be extended to odd dimensions in the same way. This recipe defines the $a$-function in odd dimensions. The value of $a$ for an interacting odd-dimensional conformal field theory connectible to a free-field theory by an RG flow is defined by formula (5.30) itself.

This prescription has a predictive content. It can be checked, in principle, in the case of an $\mathrm{RG}$ flow interpolating between two free-field theories, where $a_{n}^{\mathrm{UV}}-a_{n}^{\mathrm{IR}}$ can be worked out in two independent ways: the evaluation of (5.30) and the continuation of the free-field value of $a$, for example (5.31).

There is no known odd-dimensional example of an RG flow between two free-field theories. Its existence is plausible, however, since four-dimensional situations of this type are known, for example the lower bound of the conformal window in supersymmetric QCD, which, according to non-Abelian electric-magnetic duality [25], is manifestly free in a dual formulation.

\section{Conclusions}

A "pondered" notion of Euler density $\tilde{G}$, linear in the conformal factor, is naturally singled out by the radiative corrections to the trace anomaly in external gravity and is relevant to the 
phenomenon of quantum irreversibility, measured by the invariant area of the graph of the beta function between the fixed points. The existence of $\tilde{G}$ is a support to the ideas of ref. [3]. Formulas for arbitrary even dimension have been derived, and detailed calculations in six and eight dimensions have been performed to check the predictions. A natural, odd-dimensional formula has been proposed by dimensional continuation.

Our expression for $\Delta a$ can be checked order by order in perturbation theory, even when there is no interacting fixed point. Here, the $\varphi^{3}$-theory in six dimensions has been considered in detail. Complications are due to the large number of invariants, as well as the renormalization mixing between the operators $\varphi^{3}$ and $\varphi^{2}$. In higher dimensions it should be possible to test our ideas using higher-derivative renormalizable field theories as a laboratory. We recall, anyway, that the idea of quantum irreversibility is meaningful in complete generality, even if the theory is non-renormalizable, provided that the intrinsic RG running of the coupling constants is isolated (in a way to be uncovered) from the spurious effects of dimensioned parameters [3]. The inequality $a_{\mathrm{UV}} \geq a_{\mathrm{IR}}$ might be violated, since higher-derivative theories are not unitary, but formula (5.30) should hold, at least if suitable positivity restrictions are imposed on the classical action.

\section{Appendix}

I report in this appendix some useful formulas, starting from the expressions of the curvature tensors in a conformally flat metric,

$$
\begin{gathered}
g_{\mu \nu}=\mathrm{e}^{2 \phi} \delta_{\mu \nu} \quad \Gamma_{\mu \nu}^{\rho}=\delta_{\nu}^{\rho} \partial_{\mu} \phi+\delta_{\mu}^{\rho} \partial_{\nu} \phi-\delta_{\mu \nu} \partial^{\rho} \phi \\
R=-2(n-1) \mathrm{e}^{-2 \phi}\left[\square \phi+\frac{n-2}{2}\left(\partial_{\mu} \phi\right)^{2}\right], \\
R_{\mu \nu}=-(n-2) \partial_{\mu} \partial_{\nu} \phi-\delta_{\mu \nu} \square \phi+(n-2) \partial_{\mu} \phi \partial_{\nu} \phi-(n-2) \delta_{\mu \nu}\left(\partial_{\alpha} \phi\right)^{2} . \\
\square_{\text {cov }} S=\mathrm{e}^{-2 \phi}\left(\square+(n-2) \partial^{\alpha} \phi \partial_{\alpha}\right) S, \quad S=\text { scalar. }
\end{gathered}
$$

The meaning of the symbol $\square$ (covariant or flat-space d'Alembertian) is clear from the context in which it is used. The conformal variations needed in the paper are

$$
\begin{aligned}
& \delta R=-2(n-1) \square \delta \phi-2 R \delta \phi, \quad \delta R_{\mu \nu}=-(n-2) \nabla_{\mu} \nabla_{\nu} \delta \phi-g_{\mu \nu} \square \delta \phi, \\
& \delta \int R^{3}=(n-6) R^{3}-6(n-1) \square R^{2} \\
& \delta \int R R_{\mu \nu} R^{\mu \nu}=(n-6) R R_{\mu \nu} R^{\mu \nu}-\frac{n+2}{2} \square R^{2}-2(n-1) \square\left(R_{\mu \nu} R^{\mu \nu}\right)-2(n-2) \nabla_{\mu}\left(R^{\mu \nu} \nabla_{\nu} R\right) \\
& \delta \int R_{\mu \nu} R^{\nu \rho} R_{\rho}^{\mu}=(n-6) R_{\mu \nu} R^{\nu \rho} R_{\rho}^{\mu}+\frac{3}{4} \frac{n-2}{n-1} \square R^{2}-\frac{3}{2} n \square\left(R_{\mu \nu} R^{\mu \nu}\right)-\frac{3}{2} \frac{n(n-2)}{n-1} \nabla_{\mu}\left(R^{\mu \nu} \nabla_{\nu} R\right) \\
& \delta \int R \square R=(n-6) R \square R-\frac{n-2}{2} \square R^{2}-4(n-1) \square^{2} R . \\
& \delta \int \mathrm{G}_{6}=(n-6) \mathrm{G}_{6} .
\end{aligned}
$$


We have used (1.6) systematically. Here $\delta=\frac{1}{\sqrt{g}} \frac{\delta}{\delta \phi}, \phi$ denoting the conformal factor of the metric.

The renormalized operator $\left[\varphi^{3}\right]$ is obtained starting from the finite insertions of $\frac{\partial S}{\partial \lambda}$ in correlators. We describe here some relevant aspects of the procedure.

There is an important preliminary observation to make. When taking the $\lambda$-derivative of the action $S$, we can omit any finite term and keep just the poles. Indeed, finite additional contributions to $\left[\varphi^{3}\right]$ (having a correct power expansion in $\lambda$ ) do not affect the result that we need, since they appear multiplied by $n-6$ in $\Theta$.

Moreover, we have to omit finite terms such as $\frac{\partial a}{\partial \lambda} \mathrm{G}_{6}, \frac{\partial b}{\partial \lambda} R R_{\mu \nu} R^{\mu \nu}$, etc., but for a different reason. Although they are finite (and therefore do not change the pole part of the renormalized operator), the expressions $\frac{\partial a}{\partial \lambda}, \frac{\partial b}{\partial \lambda}$, etc., do not have an ordinary power expansion in $\lambda$ [17] and cannot appear in the definition of $\left[\varphi^{3}\right]$. The reason is readily explained. From (4.6) we see that $a$ satisfies the equation

$$
\beta \frac{\partial a}{\partial \lambda}+\frac{n-6}{2 \lambda} \frac{\partial}{\partial \lambda}\left(\lambda^{2} a\right)=\beta_{a}(\lambda) .
$$

A power expansion for $\beta_{a}(\lambda)$, assured by our arguments, excludes a power expansion for $a$. This is not a contradiction in the derivation, but an important property of the functions appearing in the induced effective action for the external gravitational background. The expressions in question do not appear in $\Theta$, nor in the induced action projected onto conformally flat metrics.

A more direct explanation of this matter is obtained by considering (6.34 for $n=6$ : we see that $\frac{\partial a}{\partial \lambda}$ contains a denominator $1 / \beta$ and we run into the problems described in the introduction. Such terms cannot appear in the expression of $\left[\varphi^{3}\right]$. Once this observation is kept in mind, there is no further obstruction to correctly identify the renormalized operator $\left[\varphi^{3}\right]$.

We have therefore, using the $\mathrm{RG}$ equations of section 4.2 ,

$$
\begin{aligned}
& \frac{\partial \lambda_{\mathrm{B}}}{\partial \lambda}= \frac{1}{2} \frac{n-6}{\widehat{\beta}} \lambda_{\mathrm{B}}, \quad \frac{\partial \ln Z_{1}}{\partial \lambda}=\frac{\gamma}{\widehat{\beta}} \\
& \frac{\partial \eta_{\mathrm{B}}}{\partial \lambda}=-\frac{1}{\widehat{\beta}}\left(\beta_{\eta}+\delta \eta\right) Z_{2}^{-1}, \quad \frac{\partial a_{\mathrm{B}}}{\partial \lambda} \rightarrow \mu^{n-6} \frac{\partial L_{a}}{\partial \lambda}=-\frac{n-6}{\widehat{\beta}} \mu^{n-6}\left(L_{a}+\frac{\beta_{a}}{n-6}\right), \\
& \frac{\partial(b, c, d)_{\mathrm{B}}}{\partial \lambda} \rightarrow-\mu^{n-6} \frac{1}{\widehat{\beta}}\left[(n-6)\left(L_{b, c, d}+\eta L_{\kappa^{(1,2,3)}}+\eta^{2} L_{\Lambda^{(1,2,3)}}+\eta^{3} L_{\Sigma^{(2)}}\right)\right. \\
&+\beta_{b, c, d}+\beta_{\eta} L_{\kappa^{(1,2,3)}}+\eta\left(\beta_{\kappa^{(1,2,3)}}+2 \beta_{\eta} L_{\Lambda^{(1,2,3)}}+\delta L_{\kappa^{(1,2,3)}}\right) \\
&\left.+\eta^{2}\left(\beta_{\Lambda^{(1,2,3)}}+3 \beta_{\eta} L_{\Sigma^{(2)}}+2 \delta L_{\Lambda^{(1,2,3)}}\right)+\eta^{3}\left(\beta_{\Sigma^{(2)}}+3 \delta L_{\Sigma^{(2)}}\right)\right]
\end{aligned}
$$

This analysis fixes only the integrated renormalized operator and so we can add arbitrary local terms, finite or divergent, which have to be fixed by requiring that $\Theta$ be finite and by studying the renormalization-group equation of $\left[\varphi^{3}\right]$. The possible local terms are (3.5) plus $\square\left[\varphi^{2}\right]$. We write their coefficients as shown in eq. (4.7).

Finally, the renormalized operator $\frac{1}{3 !} \mu^{-(n-6) / 2}\left[\varphi^{3}\right]$ turns out to be equal to $\frac{(n-6)}{\hat{\beta}}$ times

$$
\frac{1}{2} \frac{\lambda_{\mathrm{B}}}{3 !} \varphi_{\mathrm{B}}^{3}-\frac{1}{2} \frac{\beta_{\eta}+\eta \delta}{n-6} R\left[\varphi^{2}\right]+\frac{\gamma}{n-6}[\mathrm{E}]-\frac{e+L_{e}}{n-6} \square\left[\varphi^{2}\right]-\mu^{n-6}\left[\left(L_{a}+\frac{\beta_{a}}{n-6}\right) \mathrm{G}_{6}\right.
$$




$$
\begin{gathered}
+\left(L_{b, c, d}+\eta L_{\kappa^{(1,2,3)}}+\eta^{2} L_{\Lambda^{(1,2,3)}}+\eta^{3} L_{\Sigma^{(2)}}\right. \\
\left.\quad+\frac{\beta_{b, c, d}+\eta \beta_{\kappa^{(1,2,3)}}+\eta^{2} \beta_{\Lambda^{(1,2,3)}}+\eta^{3} \beta_{\Sigma^{(2)}}}{n-6}\right)\left(R R_{\mu \nu} R^{\mu \nu}, R^{3}, R \square R\right) \\
+\frac{1}{n-6}\left(f^{(1,2,3,4)}+\eta g^{(1,2,3,4)}+\eta^{2} h^{(1,2,3)}\right. \\
\left.\left.\quad+L_{f^{(1,2,3,4)}}+\eta L_{g^{(1,2,3,4)}}+\eta^{2} L_{h^{(1,2,3)}}\right)\left(\square\left(R_{\mu \nu} R^{\mu \nu}\right), \square R^{2}, \square^{2} R, \nabla_{\mu}\left(R^{\mu \nu} \nabla_{\nu} R\right)\right)\right]
\end{gathered}
$$

One can check that the operator $\frac{1}{3 !}\left[\varphi^{3}\right] \mu^{-(n-6) / 2}$ defined by this formula has a correct power expansion in $\lambda$.

Finiteness of $\Theta$, formula (4.8), fixes the pole series of the total-derivative terms in $\left[\varphi^{3}\right]$. We obtain

$$
\begin{aligned}
L_{e} & =-(n-1) L_{\eta}, \\
L_{f^{(1)}} & =-2(n-1)\left(L_{b}-L_{\eta} L_{\kappa^{(1)}}\right), \quad L_{g^{(1)}}=4(n-1) L_{\eta} L_{\Lambda^{(1)}}, \quad L_{h^{(1)}}=2(n-1) L_{\Lambda^{(1)}}, \\
L_{f^{(2)}} & =-\frac{1}{2}(n+2) L_{b}-6(n-1) L_{c}-\frac{n-2}{2} L_{d}+2(n-1) L_{\eta} L_{\kappa^{(2)}}, \\
L_{g^{(2)}} & =-\frac{1}{2}(n+2) L_{\kappa^{(1)}}-4(n-1) L_{\kappa^{(2)}}-\frac{n-2}{2} L_{\kappa^{(3)}}+4(n-1) L_{\eta} L_{\Lambda^{(2)}}, \\
L_{h^{(2)}} & =-\frac{1}{2}(n+2) L_{\Lambda^{(1)}}-2(n-1) L_{\Lambda^{(2)}}-\frac{n-2}{2} L_{\Lambda^{(3)}}+6(n-1) L_{\eta} L_{\Sigma^{(2)}}, \\
L_{f^{(3)}} & =-2(n-1)\left(2 L_{d}-L_{\eta} L_{\kappa^{(3)}}\right), \quad L_{g^{(3)}}=-2(n-1)\left(L_{\kappa^{(3)}}-2 L_{\eta} L_{\Lambda^{(3)}}\right), \\
L_{f^{(4)}} & =-2(n-2) L_{b}, \quad L_{g^{(4)}}=-2(n-2) L_{\kappa^{(1)}}, \quad L_{h^{(4)}}=-2(n-2) L_{\Lambda^{(1)}},
\end{aligned}
$$

Next, we have to relate the finite functions $f, g, h$ to the other finite functions. This can be done by studying the renormalization-group properties of the operator $\left[\varphi^{3}\right]$. We observe that $\hat{\beta}^{-1} D \hat{\beta}$ produces finite quantities when acting on finite quantities. We therefore consider

$$
\hat{\beta}^{-1} D\left(\hat{\beta} \frac{1}{3 !} \mu^{-(n-6) / 2}\left[\varphi^{3}\right]\right)=(\text { finite }),
$$

re-express $\left[\varphi^{3}\right]$ in terms of $\Theta$, and use $D \Theta=0$. Moreover we use

$$
D\left[\varphi^{2}\right]=-\delta\left[\varphi^{2}\right]-2 \mu^{n-6}\left(\beta_{\kappa^{(1,2,3)}}+2 \eta \beta_{\Lambda^{(1,2,3)}}+3 \eta^{2} \beta_{\Sigma^{(2)}}\right)\left(R_{\mu \nu} R^{\mu \nu}, R^{2}, \square R\right),
$$

which can be found by direct inspection of (4.2). The result is

$$
\begin{aligned}
(n-1) \beta_{\eta}=\delta e, \quad 2(n-1) \beta_{b}-2 e \beta_{\kappa^{(1)}}+\beta_{\eta} g^{(1)} & =2 \frac{\beta}{\lambda} f^{(1)}, \\
-4 e \beta_{\Lambda^{(1)}}+2 \beta_{\eta} h^{(1)}=\left(2 \frac{\beta}{\lambda}-\delta\right) g^{(1)}, \quad-(n-1) \beta_{\Lambda^{(1)}} & =\left(\frac{\beta}{\lambda}-\delta\right) h^{(1)}, \\
\frac{n+2}{2} \beta_{b}+6(n-1) \beta_{c}+\frac{n-2}{2} \beta_{d}-2 e \beta_{\kappa^{(2)}}+\beta_{\eta} g^{(2)} & =2 \frac{\beta}{\lambda} f^{(2)}, \\
\frac{n+2}{2} \beta_{\kappa^{(1)}}+4(n-1) \beta_{\kappa^{(2)}}+\frac{n-2}{2} \beta_{\kappa^{(3)}}-4 e \beta_{\Lambda^{(2)}}+2 \beta_{\eta} h^{(3)} & =\left(2 \frac{\beta}{\lambda}-\delta\right) g^{(2)},
\end{aligned}
$$




$$
\begin{aligned}
& \frac{n+2}{2} \beta_{\Lambda^{(1)}}+2(n-1) \beta_{\Lambda^{(2)}}+\frac{n-2}{2} \beta_{\Lambda^{(3)}}-6 e \beta_{\Sigma^{(2)}}+\delta \beta_{\Lambda^{(3)}}=2\left(\frac{\beta}{\lambda}-\delta\right) h^{(2)}, \\
& 4(n-1) \beta_{d}-2 e \beta_{\kappa^{(3)}}+\beta_{\eta} g^{(3)}=2 \frac{\beta}{\lambda} f^{(3)}, \quad 2(n-1) \beta_{\kappa^{(3)}}-4 e \beta_{\Lambda^{(3)}}=\left(2 \frac{\beta}{\lambda}-\delta\right) g^{(3)} \text {, } \\
& 2(n-2) \beta_{b}+\beta_{\eta} g^{(4)}=2 \frac{\beta}{\lambda} f^{(4)}, \quad 2(n-2) \beta_{\kappa^{(1)}}+2 \beta_{\eta} h^{(4)}=\left(2 \frac{\beta}{\lambda}-\delta\right) g^{(4)}, \\
& (n-2) \beta_{\Lambda^{(1)}}=\left(\frac{\beta}{\lambda}-\delta\right) h^{(4)} .
\end{aligned}
$$

\section{References}

[1] S. Ferrara, R. Gatto and A. Grillo, Positivity constraints on anomalous dimensions, Phys. Rev. D 9 (1974) 3564.

[2] O. Nachtmann, Positivity constraints for anomalous dimensions, Nucl. Phys. B 63 (1973) 237.

[3] D. Anselmi, Anomalies, unitarity and quantum irreversibility, Ann. Phys. (NY) 276 (1999) 361 and hep-th/9903059.

[4] S.L. Adler, J.C. Collins and A. Duncan, Energy-momentum-tensor trace anomaly in spin 1/2 electrodynamics, Phys. Rev. D15 (1977) 1712.

[5] N.K. Nielsen, The energy momentum tensor in a nonabelian quark gluon theory, Nucl. Phys. B 120 (1977) 212.

[6] J.C. Collins, A. Duncan and S.D. Joglekar, Trace and dilatation anomalies in gauge theories, Phys. Rev. D 16 (1977) 438.

[7] A.B. Zamolodchikov, "Irreversibility" of the flux of the renormalization group in a 2D field theory, JETP Lett. 43 (1986) 730.

[8] D.R. Karakhayan, R.P. Manvelyan and R.L. Mkrtchyan, Trace anomalies and cocycles of Weyl and diffeomorphisms groups, Mod. Phys. Lett. A 11 (1996) 409 and hep-th/9411068.

[9] R.J. Riegert, A non-local action for the trace anomaly, Phys. Lett. B 134 (1984) 56.

[10] G. 't Hooft, Can we make sense out of "quantum chromodynamics"?, in The whys of subnuclear physics, Erice Lectures, Ed. by A. Zichichi, Plenum Press, New York, 1977.

[11] D. Anselmi, Central functions and their physical implications, JHEP 05 (1998) 005 and hep-th/9702056.

[12] D. Anselmi, D.Z. Freedman, M.T. Grisaru and A.A. Johansen, Non-perturbative formulas for central functions in supersymmetric theories, Nucl. Phys. B526 (1998) 543 and hepth/9708042. 
[13] S. Coleman and R. Jackiw, Why dilatation does not generate dilatations, Ann. Phys. (NY) 67 (1971) 552.

[14] M.T. Grisaru, B. Milewski and D. Zanon, The supercurrent and the Adler-Bardeen theorem, Nucl. Phys. B 266(1986) 589.

[15] N. Arkani-Hamed, G.F. Giudice, M.A. Luty and R. Rattazzi, Supersymmetry-breaking loops from analytic continuation into superspace, Phys. Rev. D58 (1998) 115005 and hep$\mathrm{ph} / 9803290$.

[16] T. Arakelyan, D. R. Karakhanyan, R. Manvelyan and R. Mkrtchyan, Trace anomalies and cocycles of Weyl group, Phys. Lett. B 353 (1995) 52.

[17] S.J. Hathrell, Trace anomalies and $\lambda \phi^{4}$ theory in curved space, Ann. Phys. (NY) 139 (1982) 136.

[18] S.J. Hathrell, Trace anomalies and QED in curved space, Ann. Phys. (NY) 142 (1982) 34.

[19] D. Anselmi, More on the subtraction algorithm, Class. and Quantum Grav. 12 (1995) 319 and hep-th/9407023.

[20] S. Ichinose and N. Ikeda, Weyl anomaly in higher dimensions and Feynman rules in coordinate space, hep-th/9810256.

E.J Copeland and D.J. Toms, Class. and Quantum. Grav. 3 (1986) 431.

[21] J.L. Cardy, Is there a $c$-theorem in four dimensions? Phys. Lett. B 215 (1988) 749.

[22] O.F. de Alcantara Bonfim, J.E. Kirkham and A.J. McKane, Critical exponents to order $\varepsilon^{3}$ for $\phi^{3}$ models of critical phenomena in $6-\varepsilon$ dimensions, J. Phys. A: Math. Gen. 13 (1980) L247.

[23] L. Bonora, P. Pasti and M. Bregola, Weyl cocycles, Class. and Quantum Grav. 3 (1986) 635

S. Deser and A. Schwimmer, Phys. Lett. B 309 (1993) 279 and hep-th/9302047.

[24] D. Anselmi, Theory of higher spin tensor currents and central charges, Nucl. Phys. B 541 (1999) 323 and hepth/9808004.

[25] N. Seiberg, Electric-magnetic duality in supersymmetric non-Abelian gauge theories, Nucl. Phys. B435 (1995) 129. 